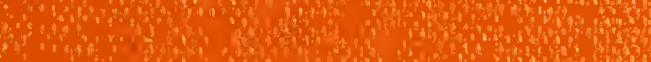






NBSIR 76-1050

Tornado-Borne Missile Speeds

Emil Simiu

Institute for Applied Technology

National Bureau of Standards

Washington, D. C. 20234

and

Martin Cordes

Institute for Basic Standards

National Bureau of Standards

Washington, D. C. 20234

April 1976

Final

Prepared for

United States Nuclear Regulatory Commission

Washington, D. C. 20555 



\section{TORNADO-BORNE MISSILE SPEEDS}

\section{Emil Simiu}

Institute for Applied Technology

National Bureau of Standards

Washington, D. C. 20234

and

Martin Cordes

Institute for Basic Standards

National Bureau of Standards

Washington, D. C. 20234

\section{April 1976}

Final

Prepared for

United States Nuclear Regulatory Commission

Washington, D. C. 20555

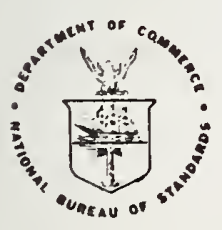

U.S. DEPARTMENT OF COMMERCE, Elliot L. Richardson, Socrotay 

Tornado - Borne Missile Speeds

Emil Simiu and Martin Cordes

At the request of the United States Nuclear Regulatory Commission (NRC) the National Bureau of Standards (NBS) has carried out an independent investigation into the question of tornado-borne missile speeds, with a view to assisting NRC in identifying pertinent areas of uncertainty and in estimating credible tornado-borne missile speeds - within the limitations inherent in the present state of the art. The investigation consists of two parts: (1) a study, covered in this report, in which a rational model for the missile motion is proposed, and numerical experiments are carried out corresponding to various assumptions on the initial conditions of the missile motion, the structure of the tornado flow, and the aerodynamic properties of the missile; (2) a theoretical and experimental study of tornado-borne missile aerodynamics, conducted by Colorado State University (CSU) under contract with NBS, to be covered in a separate report by CSU. In the present report, the factors affecting missile motion, and their influence upon such motion, are examined. Information is provided on a computer program developed for calculating missile speeds. Maximum speeds for a number of specified potential tornado-borne missiles are presented, corresponding to a set of assumptions believed by the writers to be reasonable for design purposes. It is pointed out that higher speeds are conceivable if it is assumed that certain circumstances, examined in the body of the report, will obtain. It is the judgment of the writers that the probabilities of occurrence of such higher speeds for any given tornado strike are low. More than qualitative estimates of such probabilities are, however, beyond the scope of this investigation.

KEY WORDS: Missiles; nuclear engineering; structural engineering; tornadoes; wind.

\section{ACKNOWLEDGMENTS}

The writers wish to acknowledge useful comments and suggestions by T.W. Reichard and J.R. Shaver, of the Center for Building Technology, and J.F. Costello, of the Nuclear Regulatory Commission. 



\section{$\underline{\text { Page }}$}

Abstract

Acknowledgements

List of Symbols

vi

List of Figures

viii

List of Tables

viii

1. Introduction

2. Model for the Tornado-Borne Missile Motion

3. Computer Program for Calculating Tornado-Borne

Missile Trajectories and Velocities

4. Numerical Computations

5. Sensitivity of Calculated Results to Changes in the

Assumed Initial Conditions or in the Assumed Tornado

Wind Speed Model

5.1 Changes in the Assumed Initial Position of the Missile

5.2 Changes in the Assumed Initial Velocity of the Missile

5.3 Changes in the Assumed Tornado Wind Speed Model

5.3.1 Tornado Described by Eqs. 5 through 9 with $v_{T}=0$.

5.3.2 Tornado Vortex Model Based on Data Obtained During the Dallas Tornado of April 2, 1957

6. Aerodynamic Forces

7. Speeds of Selected Tornado-Borne Missiles

8. Conclusions

9. References

APPENDIX A - Equations of Motion.

APPENDIX B - Analytic Solution to the Equations of Motion for a Uniform Wind Field. 


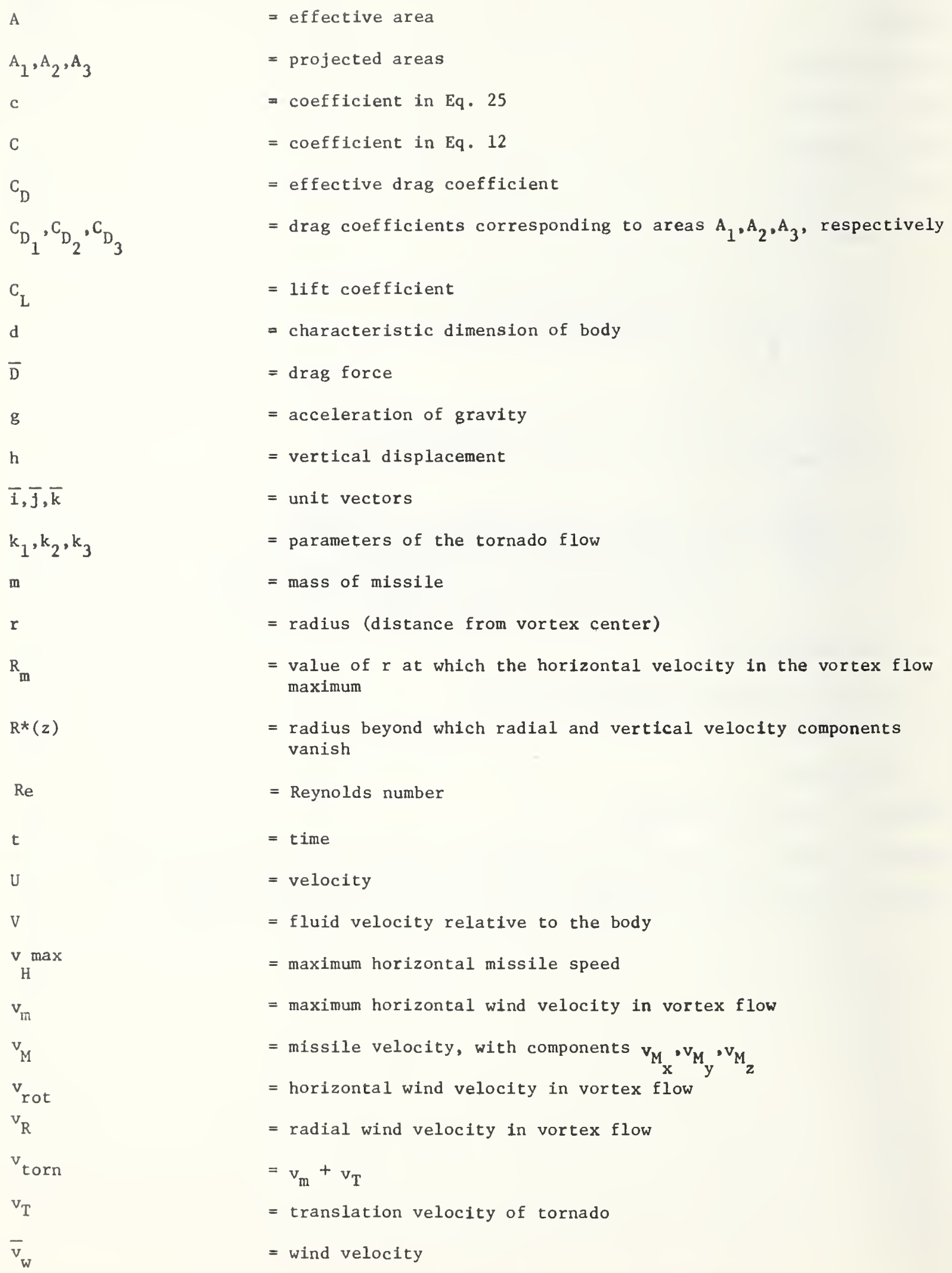

A

= effective area

= projected areas

$=$ coefficient in Eq. 25

= coefficient in Eq. 12

$=$ effective drag coefficient

$=$ drag coefficients corresponding to areas $A_{1}, A_{2}, A_{3}$, respectively

$=$ lift coefficient

- characteristic dimension of body

= drag force

= acceleration of gravity

= vertical displacement

= unit vectors

= parameters of the tornado flow

= mass of missile

= radius (distance from vortex center)

$=$ value of $r$ at which the horizontal velocity in the vortex flow maximum

= radius beyond which radial and vertical velocity components vanish

$=$ Reynolds number

$=$ time

= velocity

= fluid velocity relative to the body

$=$ maximum horizontal missile speed

= maximum horizontal wind velocity in vortex flow

= missile velocity, with components $v_{M_{x}} \cdot v_{M_{y}}, v_{M_{2}}$

= horizontal wind velocity in vortex flow

= radial wind velocity in vortex flow

$=\mathrm{v}_{\mathrm{m}}+\mathrm{v}_{\mathrm{T}}$

= translation velocity of tornado

= wind velocity 


$\begin{array}{ll}v_{z} & =\text { vertical wind velocity } \\ v_{\theta} & =\text { tangential wind velocity in vortex flow } \\ v_{\theta} & =\text { maximum tangential velocity } \\ \mathrm{w} & =\text { weight of missile } \\ \mathrm{x} & =\text { coordinate axis } \\ \mathrm{y} & =\text { coordinate axis } \\ \mathrm{z} & =\text { coordinate axis } \\ \alpha & =\text { angular displacement } \\ v & =\text { kinematic viscosity } \\ 0 & =\text { air density }\end{array}$


Figure 3 Variation of Maximum Horizontal Missile Speed

\section{LIST OF TABLES}

1. Values of $v_{T}, v_{m}$ and $R_{m}$ Used in Numerical Calculations

2. Maximum Horizontal Missile Speeds, $v_{H}^{\text {max }}$, (m/s) Corresponding to Various Initial Positions.

3. Maximum Horizontal Missile Speeds, $v_{H}^{\text {Iudx }},(\mathrm{m} / \mathrm{s})$ Corresponding to Initial Elevations $z(0)=10 \mathrm{~m}, z(0)=20 \mathrm{~m}, z(0)=40 \mathrm{~m}\left(\mathrm{C}_{\mathrm{D}} \mathrm{A} / \mathrm{m}=0.001\right)$.

4. Maximum Horizontal Missile Speeds, $v_{H}^{\text {max }}$, (m/s) Corresponding to Various Initial Velocities.

5. Maximum Horizontal Missile Speeds, $v_{H}^{\text {max }},(m / s)$ Corresponding to Various Initial Positions $\left(v_{T}=0\right)$.

6. Maximum Horizontal Missile Speeds, $v_{H}^{\max },(\mathrm{m} / \mathrm{s})$ Corresponding to Various
Parameter Values.

7. Comparison Between Calculated Speeds Based on Eqs. 13-24 and on Eqs. 5-9.

8. Characteristics and Maximum Horizontal Speeds of Selected Missiles. 


\section{INTRODUCTION}

To ensure the safety of nuclear power plants in the event of a tornado strike it is required that, in addition to the direct action of the wind and of the moving ambient pressure field, the designer consider the impact of tornado-borne missiles, i.e., of objects moving under the action of aerodynamic forces induced by the tornado wind. It is, therefore, necessary that estimates be made of the speeds attained by potential missiles under tornado wind conditions specified for the design of nuclear power plants.

Several such estimates have been reported so far $[1,2,3,4,5,6,7]$. In certain instances the differences between various estimates are very large; for example, the predicted speeds of a utility pole [13-in $(0.33 \mathrm{~m})$ diameter and a $35-\mathrm{ft}(10.7 \mathrm{~m})$ length] predicted by Refs. 5, 6, 1, 2, 4 and 3 are $16.5 \mathrm{~m} / \mathrm{s}, 30.5 \mathrm{~m} / \mathrm{s}, 41.2 \mathrm{~m} / \mathrm{s}, 42.7 \mathrm{~m} / \mathrm{s}, 52.2$ $\mathrm{m} / \mathrm{s}$ and $54.9 \mathrm{~m} / \mathrm{s}$, respectively. For any given missile, the kinetic energy associated with translational motion is proportional to the square of the speed; in the case of the utility pole, therefore, the ratio of the largest to the smallest of the kinetic energy estimates of Ref. 1 through Ref. 6 is greater than 10. For other missiles, the ratio varies from almost 2 in the case of a 1 -in $(2.54 \mathrm{~cm})$ diameter steel rod to over 5 in the case of a $4000 \mathrm{lb}(18,000 \mathrm{~N})$ standard automobile.

In most cases, such large discrepancies are the consequence of differences between basic assumptions used in the various estimation procedures. These assumptions include:

- the initial conditions of the problem, i.e., the initial position of the object with respect to the ground and to the tornado center, and the initial velocity of the object.

- the detailed features of the wind flow field.

- the aerodynamic characteristics of the object (which, in most cases of practical interest, is a bluff body).

Differences between the various sets of basic assumptions used in estimating tornadoborne missile velocities may be ascribed, in part, to the probabilistic nature of the problem. Indeed, for any given tornado wind field, the initial conditions constitute a set or random variables with a very large number of possible values, the choice of which is not unique. More importantly, however, such differences are a consequence of serious uncertainties regarding both the structure of the tornado flow and the aerodynamic behavior of the potential missile. 
At the request of the United States Nuclear Regulatory Commission (NRC), the National Bureau of Standards has carried out an independent investigation into the question of tornado-borne missile speeds, with a view to assisting NRC in Identifying pertinent areas of uncertainty and in estimating credible tornado-borne missile speeds--within the 1imitations inherent in the current state of the art.

The objectives of the investigation were defined as follows:

1. To select a rational model for the tornado-borne missile motion.

2. To develop a computer program based on this model, capable of computing missile trajectories and velocities for any specified initial conditions, tornado wind speed model, and assumptions regarding the drag force acting on the missile.

3. To calculate, for a specified set of initial conditions and for a specified wind speed model, the trajectories and velocities corresponding to a number of specified potential missiles.

4. To determine, in a number of representative cases, the sensitivity of the calculated results to changes in the assumed initial conditions or in the assumed tornado wind speed model.

5. To obtain and interpret information, based on wind tunnel tests, regarding the aerodynamic behavior of various potential missiles, i.e., drag coefficients for various missile motions, including motion in the tumbling mode.

6. From the results of items 3, 4 and 5, suggest credible speeds of selected potential tornado-borne missiles, compatible with the current state of the art in nuclear power plant design.

\section{MODEL FOR THE TORNADO-BORNE MISSILE MOTION}

The motion of an object may be described in general by solving a system consisting of three equations of balance of momenta and three equations of balance of moments of momenta. In the case of a bluff body, one major difficulty in writing these six equations is that the aerodynamic forcing functions are not known.

It is possible to measure in the wind tunnel aerodynamic forces and moments acting on a bluff body under static conditions for a sufficient number of positions of the body with respect to the mean direction of the flow. On the basis of such measurements, the dependence of the forces and moments on position, and corresponding aerodynamic derivatives, can be obtained. Aerodynamic forces and moments can then be calculated following the well-known pattern used in airfoll theory; for example, if an airfoil has a time-dependent vertical motion $h(t)$ in a uniform flow with velocity $U$, and if the angle of attack is $\alpha=$ const, the lift coefficient is

$$
C_{L}=\frac{\mathrm{dC}_{L}}{d \alpha}\left(\alpha+\frac{1}{U} \frac{d h}{d t}\right)
$$

This procedure for calculating aerodynamic forces and moments is valid if the quasisteady assumption [Ref. 8, p. 192] is acceptable and if the body concerned behaves 
aerodynamically like an airfoil - i.e., if the body is streamlined and if no flow separation occurs. However, in the case of unconstrained bluff bodies moving in a wind flow the validity of such a procedure remains to be demonstrated.

In the absence of a satisfactory model for the aerodynamic description of the missile as a rigid (six-degrees-of-freedom) body, it is customary to resort to the alternative of describing the missile as a material point acted upon by a drag force

$$
\overline{\mathrm{D}}=1 / 2 \rho \mathrm{C}_{\mathrm{D}} \mathrm{A} \mathrm{T}_{\mathrm{v}}-\overline{\mathrm{v}}_{\mathrm{M}} \mid\left(\overline{\mathrm{v}}_{\mathrm{W}}-\overline{\mathrm{v}}_{\mathrm{M}}\right)
$$

where $0=$ air density, $\bar{v}_{w}=$ wind velocity, $\bar{v}_{M}=$ missile velocity, A is a suitably chosen area and $C_{D}$ is the corresponding drag coefficient.

This model is reasonable if, during its motion, the missile either (a) maintains a constant or almost constant attitude with respect to the relative velocity vector $\bar{v}_{w}-$ $\bar{v}_{M}$, or (b) has a tumbling motion such that, with no significant errors, some mean value of the quantity $C_{D} A$ can be used in the expression for the $d r a g ~ \bar{D}$. The assumption of a constant body attitude with respect to the flow would be credible if the aerodynamic force were applied at all times exactly at the center of mass of the body--which is highly unlikely in the case of a bluff body in a tornado flow--, or if the body rotation induced by a non-zero aerodynamic moment with respect to the center of mass were inhibited by aerodynamic damping forces intrinsic in the body-fluid system. The question thus arises as to whether such stabilizing forces may be expected to be present.

It is of interest at this point to mention certain experimental results--obtained in studies of bridge deck aerodynamic stability--which provide useful insights into the question at hand. Consider a body restrained by four springs of equal stiffness, immersed in a horizontal flow (Fig. 1), and subjected to an impulse which produces angular oscillations $\theta(t)$ about the position of equilibrium [9]. In the case of an airfoil with a sufficiently small angle of attack so that flow separation does not occur, the aerodynamic damping, which is proportional to the quantity denoted by Hัخ in Ref. 9, is positive. This implies that the flow will contribute, along with the viscous damping inherent in the springs, to the damping out of the oscillations. On the other hand, for bluff bodies, at high velocities of the flow and for vanishing values of the spring stiffness, the aerodynamic damping is negative in the large majority of the cases tested [9]."

These results suggest that, in general, no stabilizing effect by the flow can be expected to inhibit the tumbling of bluff bodies. The assumption that potential tornadoborne missiles will tumble during their motion appears therefore to be reasonable. It will be this assumption that will be used in this work. 


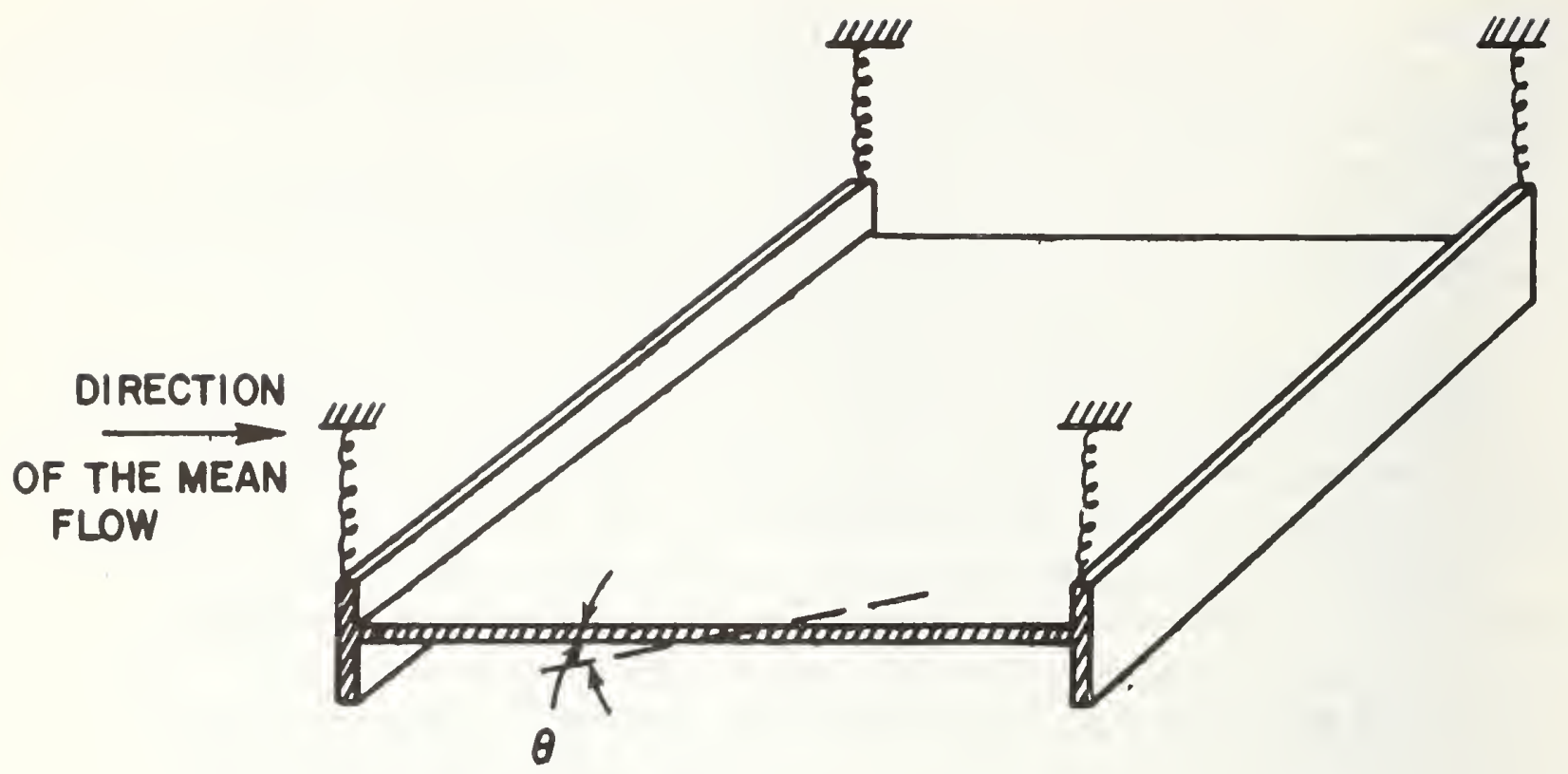

Flgure 1 Bluff Bridge Deck Section

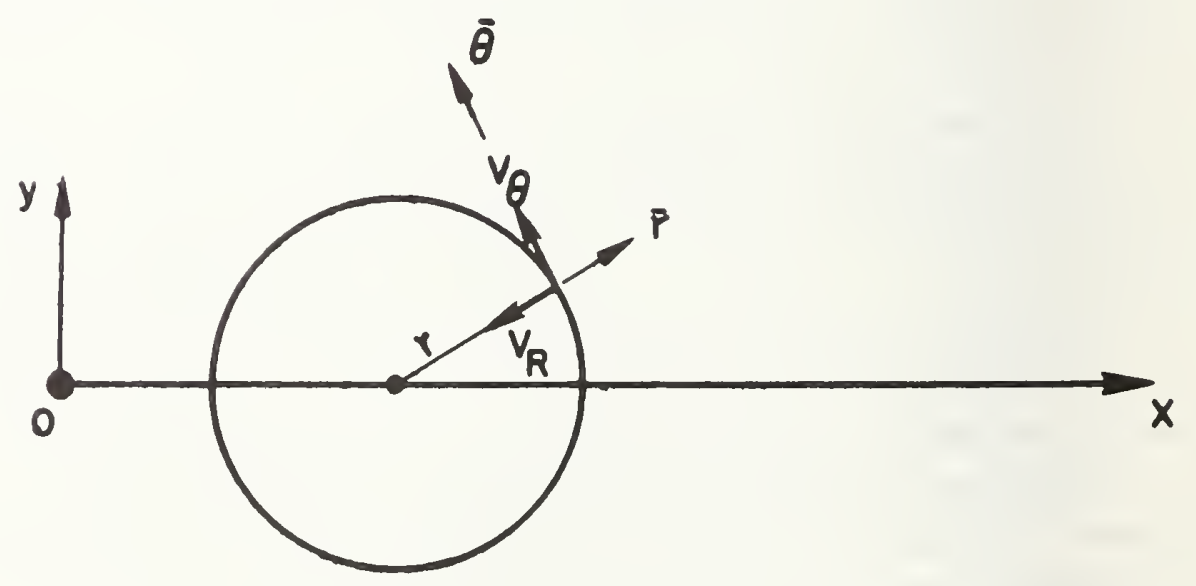

Figure 2 Notations 
Assuming that Eq. 2 is valid and that the average lift force vanishes under tumbling conditions, the motion of the missile viewed as a one-degree-of-freedom system is governed by the relation:

$$
\frac{d \bar{v}_{M}}{d t}=-1 / 2 \rho \frac{C_{D}^{A}}{m}\left|\bar{v}_{M}-\bar{v}_{W}\right|\left(\bar{v}_{M}-\bar{v}_{W}\right)-g \bar{k}
$$

where $g=$ acceleration of gravity, $\bar{k}=$ unit vector along the vertical axis and $\mathrm{m}=\mathrm{mass}$ of missile.

It follows from Eq. 3 that for a given flow field and initial conditions, the motion depends only upon the value of the parameter $C_{D} A / m$. Throughout this work, the area, $A$, will be expressed in $\mathrm{m}^{2}$ and the mass, $\mathrm{m}$, in $\mathrm{kg}$. To transform the parameter $C_{D} A / w$, where $A$ is expressed in $f t^{2}$ and the weight in $1 \mathrm{~b}$, into the parameter $C_{D} A / m$, where $A$ is expressed in $\mathrm{m}^{2}$ and the mass, $\mathrm{m}$, in $\mathrm{kg}$, the following relations are used:

$$
\begin{aligned}
& \frac{C_{D} A}{w}=1 \frac{\mathrm{ft}^{2}}{1 b} \quad \rightarrow \quad \frac{C_{D} A}{m}=.205 \frac{\mathrm{m}^{2}}{\mathrm{~kg}} \\
& \frac{C_{D} A}{m}=1 \frac{m^{2}}{k g} \quad \rightarrow \quad \frac{C_{D} A}{w}=4.902 \frac{\mathrm{ft}^{2}}{1 b}
\end{aligned}
$$

\section{COMPUTER PROGRAM FOR CALCULATING TORNADO-BORNE MISSILE TRAJECTORIES AND VELOCITIES}

To calculate and plot trajectories and velocities of tornado-borne missiles, a computer program was developed in which the assumed models for the tornado wind field and the drag coefficients are specified by specialized subroutines (details of such models are given in subsequent sections).

In Eq. 3 the components of the missile velocity $\vec{v}_{M}$ and of the wind velocity $\vec{v}_{w}$ must be referred to an absolute frame. The wind velocity $\bar{v}_{w}$ is usually specified as a sum of two parts. The first part represents the wind velocity of a stationary tornado vortex and is referred to a cylindrical system of coordinates. The second part represents the translation velocity of the tornado vortex--or, equivalently, of the cylindrical system of coordinates--with respect to an absolute frame of reference. The transformations required to represent $\bar{v}_{W}$ in an absolute frame are derived in Appendix A and are incorporated in the computer program. Documentation on the computer program and a sample input and output are given in Appendix $C$. The program, which is written in ANSI Fortran 1anguage, may be obtained on tape from the National Technical Information Service, Springfield, Virginia 22151.

For the particular case of a parallel flow, an analytical solution to the problem of the missile trajectory can be easily obtained. This solution, which can be found in Appendix B, has been used to test the program, in which the subroutine describing the wind field was modified to represent a parallel flow. 


\section{NUMERICAL COMPUTATIONS}

It was previously noted that, for a glven flow field and for given initial conditions, the missile motion depends only upon the value of the parameter $C_{D} A / m$. In this section, numerical results will be presented which show the effect of this parameter on the maximum horizontal missile speed. The calculations are based on the following assumptlons:

1. The parameter $C_{D} A / m$ is constant during the missile flight.

2. The tornado wind field may be represented by a vortex translating with a uniform velocity $v_{T}$ along an axis denoted by $0 x\left(\right.$ Fig. 2). Let $v_{R}, v_{\theta}, v_{z}$ and $v_{\text {rot }}=\left(v_{R}^{2}\right.$ $\left.+v_{\theta}{ }^{2}\right)^{1 / 2}$ denote the radial, tangential, vertical and horizontal velocity in the vortex flow, respectively. It is assumed that

$$
\begin{array}{ll}
v_{\text {rot }}=\frac{r}{R_{m}} v_{m} & 0 \leq r \leq R_{m} \\
v_{\text {rot }}=\frac{R_{m}}{r} v_{m} & R_{m} \leq r \leq \infty
\end{array}
$$

where $v_{m}$ is the maximum horizontal velocity in the vortex flow, $r$ is the radius (distance from the vortex center) and $R_{m}$ is the value of $r$ at which this velocity is attained. Eqs. 5 and 6 are simflar to descriptions of the flow proposed in Ref. 10 (p. 135). Furthermore, it is assumed that $v_{R}=1 / 2 v_{\theta}$ and $v_{z}=0.67 v_{\theta}$, 1.e., (see Fig. 2).

$$
\begin{aligned}
& v_{R}=-\frac{1}{\sqrt{5}} v_{\text {rot }} \\
& v_{\theta}=\frac{2}{\sqrt{5}} v_{\text {rot }} \\
& v_{z}=\frac{4}{3 \sqrt{5}} v_{\text {rot }}
\end{aligned}
$$

The type of model just described is referred to as the Rankine vortex and appears to be a reasonable representation of tornado flows. Estimates based on fleld observations suggest that it is reasonable to assume--as is done in this model--that $R_{m}$ is independent of height [Ref. 10, p. 131]. 
The following values for the tornado wind field parameters were used in the calculations:

Table 1 - Values of $v_{T}, v_{m}$ and $R_{m}$ Used in Numerical Calculations

\begin{tabular}{|c|c|c|c|c|c|c|c|c|}
\hline \multirow{2}{*}{$\begin{array}{c}\text { Tornado } \\
\text { Type }\end{array}$} & \multicolumn{2}{|c|}{$\mathrm{v}_{\mathrm{T}}$} & \multicolumn{2}{|c|}{$\mathrm{v}_{\mathrm{m}}$} & $\mathrm{v}_{\mathrm{torn}}=\mathrm{v}_{\mathrm{m}}+\mathrm{v}_{\mathrm{T}}$ & \multicolumn{2}{c|}{$\mathrm{R}_{\mathrm{m}}$} \\
\cline { 2 - 9 } & $\mathrm{m} / \mathrm{s}^{*}$ & $\mathrm{mph}$ & $\mathrm{m} / \mathrm{s}^{*}$ & $\mathrm{mph}$ & $\mathrm{m} / \mathrm{s}^{*}$ & $\mathrm{mph}$ & $\mathrm{m} *$ & $\mathrm{ft}$ \\
2 & 31 & 70 & 130 & 290 & 161 & 360 & 46 & 150 \\
3 & 27 & 60 & 107 & 240 & 134 & 300 & 46 & 150 \\
4 & 22 & 50 & 85 & 190 & 107 & 240 & 46 & 150 \\
5 & 31 & 70 & 146 & 325 & 177 & 395 & 46 & 150 \\
6 & 27 & 60 & 120 & 288 & 147 & 348 & 46 & 150 \\
\hline
\end{tabular}

* Approximately

The values given in Table 1 for tornado types 1, 2, 3 are suggested in Ref. 11 as providing an acceptably low level of failure if used in the design of nuclear power plants. The values for tornado type 4, 5 and 6 were included for the purpose of studying the effect upon missile velocity of relatively small increments in the value of $\mathrm{v}_{\mathrm{m}}$.

3. The assumed initial conditions are: $x(0)=\mathrm{R}_{\mathrm{m}}, \mathrm{y}(0)=0, z(0)=40 \mathrm{~m}, \mathrm{v}_{\mathrm{Mx}}(0)=0$, $v_{M y}(0)=0, v_{M z}(0)=0$ at time $t=0$, where $x, y, z$ are the coordinates of the missile (i.e., of its center of mass) and $\mathrm{v}_{\mathrm{Mx}}, \mathrm{v}_{\mathrm{My}}, \mathrm{v}_{\mathrm{Mz}}$ are the missile velocity components along the $x, y, z$ axes (Fig. 2). Also, at time $t=0$ the center of the tornado vortex coincides with the origin 0 of the coordinate axes. The effect of assuming initial conditions different from those indicated is examined in the next section of this report.

The dependence upon the parameter $C_{D^{A}} / m$ of the maximum horizontal missile speed calculated in accordance with assumptions 1 through 3 is represented in Fig. 3 for tornado types 1 through 6 as defined in Table 1. In Fig. $3 v_{\text {torn }}=v_{T}+v_{m}$ (Table 1).

\section{SENSITIVITY OF CALCULATED RESULTS TO CHANGES IN THE ASSUMED INITIAL CONDITIONS OR IN THE ASSUMED TORNADO WIND SPEED MODEL}

\subsection{Changes in the Assumed Initial Position of the Missile.}

For flows with $v_{m}=146 \mathrm{~m} / \mathrm{s}, \mathrm{R}_{\mathrm{m}}=46 \mathrm{~m}$, and $\mathrm{v}_{\mathrm{T}}=31 \mathrm{~m} / \mathrm{s}$, the maximum horizontal speeds of missiles with $C_{D} A / m=0.001$ and $C_{D} A / m=0.01$ were calculated using the initial positions shown in Table 2. Except for the initial positions, the results of Table 2 are based on the assumptions described in the preceding section. It is seen from Table 2 that 
Table 2 - Maximum Horizontal Missile Speeds, $v_{H}^{\max },(\mathrm{m} / \mathrm{s})$

Corresponding to Various Initial Positions

\begin{tabular}{|c|c|c|c|c|c|}
\hline \multicolumn{4}{|c|}{ Initial Position } & \multicolumn{2}{|c|}{$C_{D} A / m$} \\
\hline (1) & (2) & (3) & (4) & (5) & $(6)$ \\
\hline & & $x(0)$ & $y(0)$ & 0.001 & 0.01 \\
\hline & & \multicolumn{2}{|c|}{ (meters) } & & \\
\hline (a) & $\mathrm{O}$ & 46 & 0 & 10 & 65 \\
\hline (b) & $\rightarrow$ & 23 & 0 & 18 & 93 \\
\hline (c) & O. & 69 & 0 & 9 & 48 \\
\hline (d) & $O \rightarrow$ & -46 & 0 & 18 & 82 \\
\hline (e) & $0 \rightarrow$ & 0 & 46 & 16 & 68 \\
\hline (f) & $0 \rightarrow$ & 0 & 23 & 20 & 84 \\
\hline (g) & Q $\rightarrow$ & 0 & -23 & 35 & 50 \\
\hline (h) & $G$ & 0 & -46 & 54 & 70 \\
\hline
\end{tabular}

1. Arrows in column (2) represent direction of translation velocity $\mathrm{v}_{\mathrm{T}}$.

2. Assumed elevation of missile at time $t=0: z(0)=40 \mathrm{~m}$ in all cases.

Table 3 - Maximum Horizontal Missile Speeds, $v_{H}^{\max }$, (m/s) Corresponding to

Initial Elevations $z(0)=10 \mathrm{~m}$,

$z(0)=\frac{\text { In and } z(0)}{20 \mathrm{~m} \text { an }(\mathrm{C} \mathrm{A} / \mathrm{m}=0.001)}$

\begin{tabular}{|c|c|c|c|c|c|c|c|}
\hline \multicolumn{5}{|c|}{ Initial Position } & \multicolumn{3}{|c|}{$z(0)$} \\
\hline (1) & (2) & & (3) & (4) & (5) & (6) & (7) \\
\hline & & & $x(0)$ & $y(0)$ & $10 \mathrm{~m}$ & $20 \mathrm{~m}$ & $40 \mathrm{~m}$ \\
\hline & & \multicolumn{3}{|c|}{ (meters) } & & & \\
\hline (a) & 0 & $\rightarrow$ & 46 & 0 & 6 & 7 & 10 \\
\hline (b) & ○ & $\rightarrow$ & 23 & 0 & 2 & 8 & 18 \\
\hline (c) & ○. & $\rightarrow$ & 69 & 0 & 9 & 9 & 9 \\
\hline (d) & 0 & $\rightarrow$ & -46 & 0 & 14 & 16 & 18 \\
\hline (e) & 0 & $\rightarrow$ & 0 & 46 & 3 & 9 & 16 \\
\hline (f) & 9 & $\rightarrow$ & 0 & 23 & 12 & 16 & 20 \\
\hline (g) & 6 & $\rightarrow$ & 0 & -23 & 19 & 29 & 35 \\
\hline (h) & Q & $\rightarrow$ & 0 & -46 & 33 & 42 & 54 \\
\hline
\end{tabular}




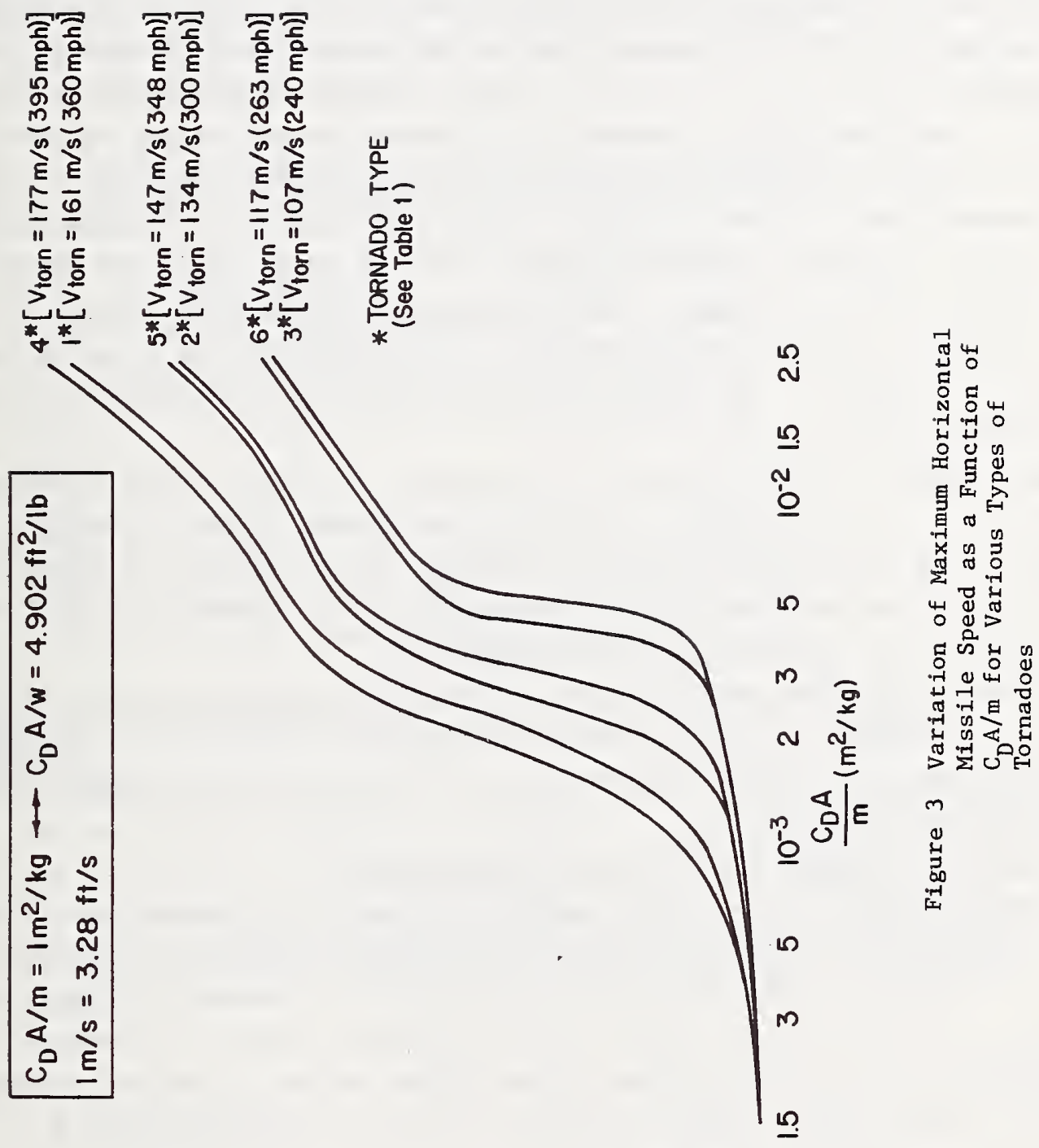

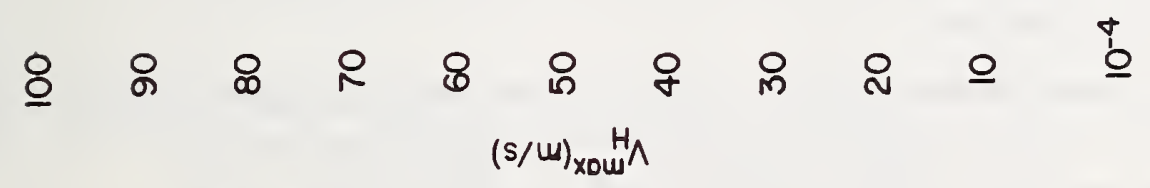


the initial position used in the calculations of the preceding section, (position (a) of Table 2) does not result in the largest possible maximum horizontal missile speeds.

It is also noted that the initial positions to which there correspond the largest possible missile speeds depend upon the value of $C_{D} A / m$. For example, for $C_{D} A / m=0.01$, that initial position is $(\bar{b})$; for $C_{D} A / m=0.001$, it is (h) [see Table 2].

As indicated in Note 2 of Table 2, the initial elevation assumed in the calculations was $z(0)=40 \mathrm{~m}$. If the weight of the missile is smaller than the upward drag induced by the vertical wind velocity component, then the calculated missile velocities at time $t$ are independent of $z(0)$. (This is the case because, in the assumed tornado model, the flow field is invariant with $\mathrm{z}$ ). However, if the missile weight exceeds the upward drag, 1.e., If the missile moves downward,--as in the case of column (5) of Table 2-- the interval between time $t=0$ and the time at which the missile hits the ground decreases as $z(0)$ decreases. Therefore, the maximum missile speed may decrease if lower values of the initial elevation $z(0)$ are assumed. Table 3 lists speeds calculated using the assumptions $z(0)=10 \mathrm{~m}$ and $z(0)=20 \mathrm{~m}$, all other values of the various parameters being the same as for column (5) of Table 2. For comparison the calculated speeds in column (5) of Table 2 were also included in Table 3.

Missile speeds were also calculated corresponding to various initial elevations $0<z(0)<10 \mathrm{~m}$. It is of interest to note that the maximum horizontal speed of a missile with $C_{D} A / m=0.001$ starting the motion from position ( $h$ ) is relatively high even for low values of the initial elevation $z(0)$. For example, for $z(0)=3 \mathrm{~m}$ and $z(0)=5 \mathrm{~m}$, the maximum missile speeds are $23 \mathrm{~m} / \mathrm{s}$ and $27 \mathrm{~m} / \mathrm{s}$, respectively.

Calculations were also carried out for the horizontal distances traveled by the missiles. For example, for $C_{D} A / M=0.001$ and the inftial position ( $h$ ), the horizontal distances corresponding to $z(0)=3 \mathrm{~m}, 5 \mathrm{~m}, 10 \mathrm{~m}, 20 \mathrm{~m}$, and $40 \mathrm{~m}$ are, approximately, $20 \mathrm{~m}, 30 \mathrm{~m}$, $50 \mathrm{~m}, 90 \mathrm{~m}$ and $160 \mathrm{~m}$, respectively.

\subsection{Changes in the Assumed Initial Velocity of the Missile.}

The results given in the preceding sections are based on the assumption that the initial velocity of the missiles is zero. If the missile is injected in the flow, for example by an explosion, this assumption is no longer valid. However, all other conditions being equal, a non-zero intial velocity does not necessarily result in a maximum missile velocity higher than that corresponding to zero initial velocity. This is illustrated in Table 4, in which type 1 tornado (see Table 1 ), and the conditions $v_{M x}(0) \neq 0$, $v_{\text {My }}(0)=0, v_{M z}(0)=0$ were assumed.

Table 4 - Maximum Horizontal Missile Speeds, $\mathrm{v}_{\mathrm{H}}^{\max }$, (m/s) Corresponding to Various Initial Velocities

\begin{tabular}{|c|c|c|c|c|c|c|c|c|c|}
\hline \multirow[b]{2}{*}{ (1) } & \multirow[b]{2}{*}{ (2) } & \multirow[b]{2}{*}{ (3) } & \multirow[b]{2}{*}{ (4) } & \multicolumn{3}{|c|}{$C_{D} A / m=0.001$} & \multicolumn{3}{|c|}{$\mathrm{C}_{\mathrm{D}} \mathrm{A}^{\prime} \mathrm{m}=0.01$} \\
\hline & & & & (5) & (6) & (7) & (8) & (9) & (10) \\
\hline & & $x(0)$ & $y(0)$ & \multicolumn{6}{|c|}{$\mathrm{v}_{\mathrm{Mx}}(0) \quad$ (meters/second) } \\
\hline & & \multicolumn{2}{|c|}{ (meters) } & 0 & 10 & 20 & 0 & 10 & 20 \\
\hline $\begin{array}{l}\text { (a) } \\
\text { (g) }\end{array}$ & & $\begin{array}{r}46 \\
0\end{array}$ & $\begin{array}{c}0 \\
-23\end{array}$ & $\begin{array}{r}8 \\
35\end{array}$ & $\begin{array}{r}9 \\
45\end{array}$ & $\begin{array}{l}20 \\
35\end{array}$ & $\begin{array}{l}62 \\
63\end{array}$ & $\begin{array}{l}58 \\
59\end{array}$ & $\begin{array}{l}53 \\
59\end{array}$ \\
\hline
\end{tabular}


5.3 Changes in the Assumed Tornado Wind Speed Model.

5.3.1 Tornado Described by Eqs. 5 through 9 with $\mathrm{v}_{\mathrm{T}}=0$.

Table 5 lists maximum horizontal missile speeds for the same parameter values and initial conditions as those used in Table 2, except that the translation velocity of the tornado vortex is zero, rather than $31 \mathrm{~m} / \mathrm{s}$. It is seen by comparing Tables 2 and 5 that the calculated speeds are, on the average, higher in the case $v_{T}=31 \mathrm{~m} / \mathrm{s}$, although for some initial positions the reverse is true.

Table 5 - Maximum Horizontal Missile Speeds, $v_{\mathrm{H}}^{\max }$, Corresponding to Various Initial Positions $\left(\mathrm{v}_{\mathrm{T}} \stackrel{\mathrm{H}}{=} 0\right)$

\begin{tabular}{|c|c|c|c|c|c|}
\hline \multicolumn{4}{|c|}{ Initial Position } & \multicolumn{2}{|c|}{$\mathrm{C}_{\mathrm{D}} \mathrm{A} / \mathrm{m}$} \\
\hline (1) & (2) & $x(0)$ & $\begin{array}{l}y(0) \\
r s)\end{array}$ & $\begin{array}{c}(2) \\
0.001\end{array}$ & $\begin{array}{l}(3) \\
0.01\end{array}$ \\
\hline $\begin{array}{l}\text { (a) } \\
\text { (b) } \\
\text { (c) } \\
\text { (d) } \\
\text { (e) } \\
\text { (f) } \\
\text { (g) } \\
\text { (h) }\end{array}$ & 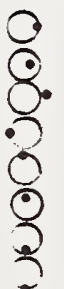 & $\begin{array}{r}46 \\
23 \\
69 \\
-46 \\
0 \\
0 \\
0 \\
0\end{array}$ & $\begin{array}{r}0 \\
0 \\
0 \\
0 \\
46 \\
23 \\
-23 \\
-46\end{array}$ & $\begin{array}{r}30 \\
7 \\
19 \\
30 \\
30 \\
8 \\
8 \\
30\end{array}$ & $\begin{array}{l}65 \\
61 \\
60 \\
65 \\
65 \\
61 \\
61 \\
65\end{array}$ \\
\hline
\end{tabular}

\subsubsection{Tornado Vortex Models Based on Data Obtained During the Dallas Tornado of}

Apri1 2, 1957 [13].

In this family of models, the velocities in the tornado vortex depend upon the quantities $\mathrm{R}_{\mathrm{m}}=$ radius of maximum tangential velocity at elevation $z=0, v_{\theta m}=$ maximum tangential velocity and $R *(z)$ = radius beyond which the radial and vertical velocity components vanish. In addition, the flow depends on three parameters $k_{1}, k_{2}, k_{3}$, as will be shown subsequently. The radius of maximum tangential velocity at elevation $z$ is denoted by $R_{m}(z)$ and is assumed to be

$$
\begin{array}{ll}
R_{m}(z)=R_{m}+k_{1} z & 0<z<60 m \\
R_{m}(z)=R_{m}+60 k_{1} & z \geq 60 m
\end{array}
$$

It is seen that the parameter $k_{1}$ is a measure of the extent to which the tornado vortex deviates from a cylindrical shape. If $k_{1}=0$ the radius of maximum tangential velocity is invariant with height, as in the model described by Eqs. 5 through 9. An assumption commonly employed in tornado-borne missile speed investigations is $k_{1}=0.45$ [see, for example, Refs. 3 and 12]. It is further assumed [3, 12]: 


$$
R^{*}(z)=C R_{m}(z)
$$

where $C$ is a coefficient which depends upon $V_{\theta m}$. In this work, it was assumed $C=2.35$ for $v_{\theta m}=130 \mathrm{~m} / \mathrm{s}, c=2.10$ for $v_{\theta_{m}}=107 \mathrm{~m} / \mathrm{s}$ and $c=1.80$ for $v_{\theta m}=85 \mathrm{~m} / \mathrm{s}$. These values are similar to those used in Ref. 3.

Let $r$ denote the distance to the center of the tornado vortex. The components of the velocity in the tornado vortex are assumed to be

$\underline{0<z<60 m}$

$$
\begin{array}{lc}
v_{R}=-k_{2} \frac{R^{*}(z)-r}{R^{*}(z)-R_{m}(z)} r & r \leq R^{*}(z) \\
v_{R}=0 & r>R^{*}(z) \\
v_{\theta}=\frac{r}{R_{m}(z)} v_{\theta m} & r \leq R_{m}(z) \\
v_{\theta}=\frac{R_{m}(z)}{r} v_{\theta m} & r>R_{m}(z) \\
v_{z}=k_{3} \frac{R^{*}(z)-r}{R^{*}(z)-R_{m}(z)} z+\frac{v_{\theta}}{3} & r<R^{*}(z) \\
v_{z}=0 & r \geq R^{*}(z)
\end{array}
$$

\section{$60 \mathrm{~m}<z<240 \mathrm{~m}$}

$$
\begin{array}{ll}
v_{R}=-k_{2} \frac{R^{*}(z)-r}{R^{*}(z)-R_{m}(z)} \times \frac{240-z}{180} & r \leq R^{*}(z) \\
v_{R}=0 & r>R^{*}(z) \\
v_{\theta}=\frac{r}{R_{m}(z)} v_{\theta m} & r \leq R_{m}(z) \\
v_{\theta}=\frac{R_{m}(z)}{r} v_{\theta m} & r>R_{m}(z) \\
v_{z}=\left(1.33-\frac{z}{180}\right)\left(k_{3} \frac{R^{*}(z)-r}{R^{*}(z)-R(z)} 60+\frac{v_{\theta}}{3}\right) \quad r<R^{*} \quad(z) \\
v_{z}=0 & r \geq R^{*}(z)
\end{array}
$$


Table 6 - Maximum Horizontal Missile Speeds $v_{H}^{\max },(\mathrm{m} / \mathrm{s})$ Corresponding to Various

Parameter Values

\begin{tabular}{|c|c|c|c|c|c|c|c|}
\hline Case & $v_{\theta_{\mathrm{m}}}$ & $\mathbf{v}_{\mathbf{T}}$ & $k_{1}$ & $\mathrm{k}_{2}$ & $k_{3}$ & $\mathrm{C}_{\mathrm{D}} \mathrm{A} / \mathrm{m}$ & $\mathrm{v}_{\mathrm{H}}^{\max }$ \\
\hline & $(\mathrm{m} / \mathrm{s})$ & $(\mathrm{m} / \mathrm{s})$ & & & & $\mathrm{m}^{2} / \mathrm{kg}$ & $(\mathrm{m} / \mathrm{s})$ \\
\hline 1 & 85 & 22 & .45 & 1 & 1 & 0.025 & $90 *$ \\
\hline 2 & 85 & 22 & .45 & 1 & 0 & 0.025 & $83 *$ \\
\hline 3 & 85 & 22 & 0 & 1 & 0 & 0.025 & 77 \\
\hline 4 & 85 & 22 & 0 & 1 & 1 & 0.025 & 79 \\
\hline 5 & 130 & 31 & .45 & 1 & 1 & 0.025 & $90 *$ \\
\hline 6 & 130 & 31 & .45 & 1 & 0 & 0.025 & $126 *$ \\
\hline 7 & 130 & 31 & 0 & 1 & 0 & 0.025 & $80 *$ \\
\hline 8 & 130 & 31 & 0 & 1 & 1 & 0.025 & $75 *$ \\
\hline 9 & 130 & 31 & .45 & 1 & 1 & 0.01 & $91 *$ \\
\hline 10 & 130 & 31 & .45 & 1 & 0 & 0.01 & 87 \\
\hline 11 & 130 & 31 & .25 & 1 & 0 & 0.01 & 73 \\
\hline 12 & 130 & 31 & 0 & 1 & 1 & 0.01 & 60 \\
\hline 13 & 130 & 31 & 0 & 1 & 0 & 0.01 & 60 \\
\hline 14 & 130 & 31 & .45 & 0 & 1 & 0.025 & 60 \\
\hline 15 & 130 & 31 & .45 & 0 & 1 & 0.01 & 36 \\
\hline
\end{tabular}

* Calculated speeds are higher at $z>60 \mathrm{~m}$. 
The most commonly used value of the parameter $k_{2}$ is unity (see, for example, Ref. 3 and 12). However, $1 t$ is by no means certain that Eqs. 13 and 19 with $k_{2}=1$ are a satisfactory model of the radial velocity $v_{R}$. Indeed, according to Ref. 10 ( $p$. 135), $v_{R}$ is negligible throughout the tornado flow except in the vicinity of $r=R_{m}(z)$. It is, therefore, of interest to examine the influence upon the calculated missile speeds of the assumption $\mathrm{v}_{\mathrm{R}}=0$. This can be done by assuming $\mathrm{k}_{2}=0$ in Eqs. 13 and 19 .

Similarly, while it is commonly assumed $k_{3}=1$, it is of interest to examine the case in which the value of the vertical velocity component $v_{z}$ is smaller than that given by Eqs. 17 and 23 with $k_{3}=1$. This can be done, for example, by assuming $k_{3}=0$.

Calculations of missile trajectories and speeds based on the assumption that the tornado vortex is described by Eqs. 13 through 24 were carried out for the 15 cases listed in Table 6. In all cases of Table 6 it was assumed $x(0)=R_{m}=46 \mathrm{~m}, y(0)=0$, $z(0)=0$. Table 6 only includes the maximum calculated horizontal missile speeds $\mathrm{v}^{\mathrm{max}}$ at elevations $z \leq 60 \mathrm{~m}$.

A discussion will now be presented of the results of Table 6 . The cases corresponding to the set of parameters $k_{1}=0.45, k_{2}=1, k_{3}=1$ which, as previously indicated, are most commonly assumed in missile speed calculations, will be examined first. It is noted that the missile speeds for cases 1, 5 and 9 of Table 6 are higher than the corresponding speeds of Fig. 3, as shown in Table 7 .

Table 7 - Comparison between Calculated Speeds Based on Eqs. 13 through 24 and on Eqs. 5 through 9

\begin{tabular}{|l|c|c|c|c|}
\hline & \multicolumn{2}{|c|}{ Table 6 } & \multicolumn{2}{c|}{ Figure 3 } \\
\hline \begin{tabular}{c|c}
$\mathrm{C}_{\mathrm{D}} \mathrm{A}$ \\
$\mathrm{m}$
\end{tabular} & Case & $\begin{array}{c}\mathrm{v}_{\mathrm{H}}^{\mathrm{max}} \\
(\mathrm{m} / \mathrm{s})\end{array}$ & $\begin{array}{c}\text { Tnrnado } \\
\text { Type }\end{array}$ & $\begin{array}{c}\mathrm{v}_{\mathrm{H}}^{\max } \\
(\mathrm{m} / \mathrm{s})\end{array}$ \\
\hline 0.025 & 1 & 90 & $3 *$ & 57 \\
0.025 & 5 & 90 & $1 *$ & 81 \\
0.01 & 9 & 91 & $1 *$ & 63 \\
\hline
\end{tabular}

* See Table 1

This can be explained as follows. Assume that $v_{R}=v_{T}=0$ and that the missile starts from an initial position $x(0)=R_{m}$. As the missile gains momentum, it tends to $f l y$ in a straight line along the direction of the tangential velocity, i.e., at larger distances from the center of the vortex. In the case of a tornado with a radius of maximum tangential speed that is constant with height, as the missile moves away from the intial position, it will be subjected to winds of lower intensity and its speed will increase more slowly. On the other hand, if the radius of maximum tangential speed increases with height, and if the elevation of the missile increases under the action of vertical wind speed components larger than the missile weight, then as the missile moves away from the center and up it continues to $\mathrm{fly}$ in zones of maximum winds and thus continues to gain momentum at a fast 
rate. This mechanism is modified only slightly in most, although not in all cases, if non-zero radial and translation velocities are present. The explanation just advanced is confirmed by comparing cases 4 to 1,8 to 5 , and 12 to 9 . In cases 4,8 and $12, k_{1}=$ 0 , i.e., the radius of maximum tangential velocity is constant, and the missile speeds are lower than in cases 1,5 , 9, in which that radius increases with height. A similar conclusion is reached by comparing cases 3 to 2, 7 to 6, 11 and 13 to 10 .

The parameter $k_{3}$, which controls the magnitude of the vertical wind velocity component, does not appear to affect the missile speeds in a uniform fashion, i.e., to the value $k_{3}$ $=0$ there correspond in certain cases lower values of the missile speed than to the value $k_{3}=1$ (e.g., case 2 vs. case 1 ); in other cases, the reverse is true (e.g., case 6 vs. case 5).

Some meteorologists have expressed the view that the radial velocity component $v_{R}$ is smaller over most of the tornado field than that described by either Eq. 7 or Eqs. 13 and 19 with $k_{2}=1[10]$. It is therefore of interest to examine the case $k_{2}=0 . \quad I t$ is seen, by comparing in Table 6 cases 14 and 15 with cases 5 and 9 , respectively, that the assumption $k_{2}=0$ results in considerably lower missile speeds than the assumption $k_{2}=$ 1. This is the case because, if $k_{2}=0$, (i.e., if $v_{R}=0$ ), the missile is ejected (i.e., it flies at larger distances from the center of the vortex and therefore, in a region of lower speeds) sooner than if $k_{2}=1$, (i.e., than if a radial velocity is present that resists the tendency of the missile to fly away from the center). This reasoning is valid for the assumed initial condition $x(0)=R_{m}, y(0)=0$. It is conceivable however, that initial conditions exist which might result in higher missile speeds for $\mathrm{k}_{2}=0$ than for $\mathrm{k}_{2}=1$.

\section{AERODYNAMIC FORCES}

In this work it was assumed that the aerodynamic force acting upon a missile is

$$
\bar{D}=1 / 2 \rho C_{D} A\left|\bar{v}_{W}-\bar{v}_{M}\right|\left(\bar{v}_{W}-\bar{v}_{M}\right)
$$

where $\rho=\operatorname{air}$ density, $\bar{v}_{W}=$ wind velocity, $\bar{v}_{M}=$ missile velocity and $C_{D} A$ is a suitably chosen quantity with the dimensions of an area. As indicated in Section 2 , Eq. 2 may be assumed to be valid under random tumbling conditions, i.e., if the motion is such that the body "presents all possible aspects to the flow,. . . the orientation of the surface elements with respect to the flow sweeps through all the possible angles" (Ref. 14) and, moreover, the angular speed of the tumbling body is sufficiently large. In this connection it may be argued that, instantaneously, lift forces do exist, and that the momentum they impart to the missile may not be negligible if the rotational motion of the missile is relatively slow. The acceleration of the missile would then no longer be parallel, at every instant, to the vector $\bar{v}_{W}-\bar{v}_{M}$ and the missile trajectory would deviate from that predicted by using the aerodynamic model implicit in Eq. 2. It is believed, however, that the effect of such deviations on the maximum speed of the tornado-borne missile is 
comparable to the effect of changes in the initial conditions of the problem such as were studjed in Section 5. For the purposes of this report, the existence of lift forces which are not taken into account in the calculations is believed not to invalidate the aerodynamic model used herein.

The value $C_{D} A$ that corresponds to tumbling conditions can, in principle, be determined experimentally. Unfortunately, little information on this topic appears to be presently available. Ref. 14 examines tumbling motions for supersonic wind conditions, while Ref. 15 contains information on tumbling under flow conditions corresponding to Mach numbers 0.5 to 3.5. Hoerner extrapolated the data of Ref. 15 to lower subsonic speeds (Ref. 16, p. 14-16, Fig. 7); according to this extrapolation, for a randomly tumbling cube the quantity $C_{D} A$ equals, approximately, the average of the products of the projected areas corresponding to "all positions statistically possible" by the respective static drag coefficients (Ref. 16, p, 14-16 and P. 13-17). An investigation into this question is currently carried out, within the framework of this project, by Colorado State University (CSU). The theoretical and experimental results of this investigation will be reported in a separate document by CSU.

In the absence of more experimental information, it appears reasonable to assume that the effective product $C_{D} A$ is given by the expression

$$
C_{D} A=C\left(C_{D_{1}} A_{1}+C_{D_{2}} A_{2}+C_{D_{3}} A_{3}\right)
$$

in which $C_{D_{1}} A_{i}(i=1,2,3)$ are products of the projected areas corresponding to the cases in whilch the principal axes of the body are parallel to the vector $\vec{v}_{W}-\vec{v}_{M}$, by the respective static drag coefficients. In Eq. 25, c is a coefficient assumed to be 0.50 for planks, rods, pipe and poles and 0.33 for the automobile.

An upper bound for the quantity $C_{D} A$ is believed to be

$$
\left(C_{D} A\right)_{u \cdot b \cdot}=C_{D_{1}} A_{1}
$$

in which $C_{D_{I}} A_{1}$ is the largest of the quantities $C_{D_{i}} A_{i}(i=1,2,3)$.

The Reynolds number is defined as

$$
\operatorname{Re}=\frac{V d}{v}
$$

where $\mathrm{V}=$ fluid velocity relative to the body, $\mathrm{d}=$ characteristic dimension of the body (in the case of a cylinder, $\mathrm{d}=$ diameter) and $\nu=$ kinematic viscosity $\left(\nu \simeq 1.5 \mathrm{x} 10^{-5}\right.$ $\mathrm{m} / \mathrm{s}$ for air). For a circular cylinder $\mathrm{Re} \simeq .67 \times 10^{5} \mathrm{Vd}$, where $\mathrm{V}$ and $\mathrm{d}$ are expressed in $\mathrm{m} / \mathrm{s}$ and $\mathrm{m}$, respectively. For $\mathrm{Re}>4 \times 10^{5}$, i.e., the Reynolds number is in the supercritical range and it may therefore be assumed, conservatively, $C_{D_{1}} \simeq 0.7$ (see Ref. 8, p. 67). In the case of the 1 inch $(2.54 \mathrm{~cm})$ rod, it may be assumed that $\operatorname{Re}$ is in the subcritical range even for velocities $V$ of the order of $100 \mathrm{~m} / \mathrm{s}$ and, therefore, that $C_{D_{1}} \simeq 1.2($ Ref. 8 ). 


\section{SPEEDS OF SELECTED POTENTIAL TORNADO-BORNE MISSILES}

In this section calculated speeds of selected potential tornado-borne missiles will be given, based on the following assumptions:

(1) The model of the tornado vortex consisting of Eqs. 5 through 9 is valid, with the parameter values corresponding to cases 1,2 and 3 of Table 1 .

(2) The initial conditions are $x(0)=R_{m}, y(0)=0, z(0)=40 m$ (for comments on the initial condition $\mathrm{z}(0)=40 \mathrm{~m}$, see $\mathrm{p} \cdot 10$ of this report $) ; \mathrm{v}_{\mathrm{Mx}}(0)=\mathrm{v}_{\mathrm{My}}(0)=\mathrm{v}_{\mathrm{Mz}}(0)$ $=0$. Assumptions (1) and (2) just described were used in calculating the values on curves 1,2 and 3 of Fig. 3.

(3) The effective product $C_{D} A$ is given by Eq. 25. The results of the calculations based on these assumptions are shown in Table 8 .

The missile speeds of Table 8 are based on a set of assumptions which, while reasonable, might in certain cases not correctly reflect the actual physical phenomenon. It follows from Sections 5 and 6 that the order of magnitude of uncertainties in the estimates of maximum missile speeds can in certain cases be as high as $50 \%$ or even more. Whether or not actual missile speeds will be higher than those listed in Table 8 depends in large measure on the extent to which the tornado flow model consisting of Eqs. 5 through 9 (the so-called Rankine vortex) is realistic. In particular, if, as suggested in Ref. 10, the radial and vertical velocity components in a tornado are actually lower than those given by this model, it could be expected--all other conditions being equal-that the predictions of Table 8 are conservative. If, on the other hand, the actual tornado flow is more closely represented by Eqs. 13 through 24 with certain unfavorable values of the parameters included in these equations, then higher missile speeds than those of Table 8 may occur, as shown in Tables 6 and 7 .

The speeds of Table 8 may also be exceeded if unfavorable initial conditions obtain. The uncertainties pertaining to the tornado flow modeling are due to the lack of reliable information; on the other hand, those pertaining to the initial conditions are a consequence of the probabilistic nature of the problem. Probabilities of occurrence may be assigned to each set of initial conditions. The probability that (a) the wind speed will reach the intensity levels of Table 1, (b) that a missile starts from a highly unfavorable set of initial conditions and (c) that it will hit a certain installation with a speed $V_{H}{ }^{m a x}$ can be.expected to be negligibly low. Such probabilities can in principle be evaluated using, for example, procedures similar to those outlined in Ref. 18. Attempts to calculate such probabilities are beyond the scope of this work. 


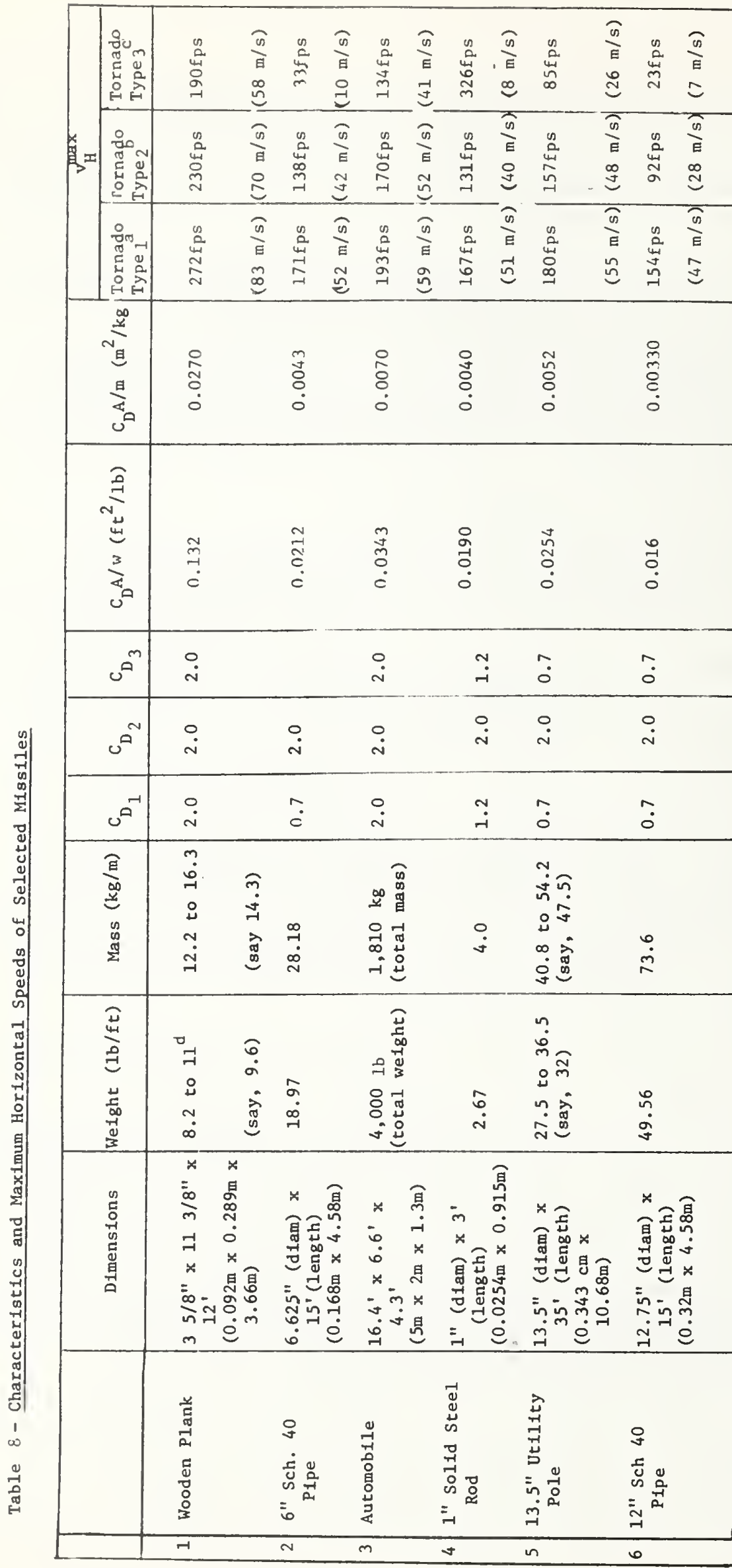

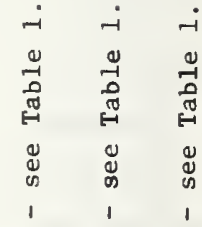

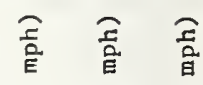

듕 \&

a n

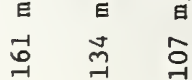

॥ "

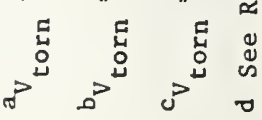


The uncertainties regarding the actual aerodynamic drag coefficients constitute another source of error. It is noted that the curves in Fig. 3 are S-shaped. Large errors in the assumed value of the quantity $\mathrm{C}_{\mathrm{D}} \mathrm{A}$ may therefore in certain cases result in considerable changes of the estimated value of $\mathrm{V}_{\mathrm{H}}^{\mathrm{max}}$ For example, if, for a 12 in Sch. 40 pipe (entry 6 in Table 8) it was assumed $C_{D} A / m=0.0066 \mathrm{~kg} / \mathrm{m}^{2}$ instead of $C_{D} A / m=0.0033$ $\mathrm{kg} / \mathrm{m}^{2}$, it follows from Fig. 3 that $\mathrm{V}_{\mathrm{H}}^{\max }=40 \mathrm{~m} / \mathrm{s}$, rather than $\mathrm{V}_{\mathrm{H}}^{\max }=7 \mathrm{~m} / \mathrm{s}$, as in Table 8. It is interesting to note, on the other hand, that as long as a change in the assumed value of $C_{D} A / m$ does not displace the point from the upper or from the lower branch of an S-shaped curve, the sensitivity of $\mathrm{V}_{\mathrm{H}}^{\mathrm{max}}$ to even considerable changes in the value of $\mathrm{C}_{\mathrm{D}} \mathrm{A} / \mathrm{m}$ is fairly small. For example, if for the 6 in Sch. 40 pipe (entry 2 in Table 8 ) it is assumed that $C_{D} A / m=0.0086$, then, for a tornado type $1, V_{H}^{\max }=61 \mathrm{~m} / \mathrm{s}$ (see $F i g$. 3), whereas to the assumption $\mathrm{C}_{\mathrm{D}} \mathrm{A} / \mathrm{m}=0.0043$ there corresponds $\mathrm{V}_{\mathrm{H}}^{\max }=52 \mathrm{~m} / \mathrm{s}$, i.e., to an error of $100 \%$ in the value of $C_{D} A / m$ there corresponds an error of only $17 \%$ in the estimated value of $v_{H}^{m a x}$.

Finally, it must be noted that the actual missiles may have properties that are more unfavorable than those listed in Table 8; for example, the case is mentioned in Ref. 19 of a beam attached, during its flight, to a portion of a carport roof which considerably increased the surface area of the missile and, therefore, the parameter $\mathrm{C}_{\mathrm{D}} \mathrm{A} / \mathrm{m}$.

\section{CONCLUSIONS}

In the preceding section calculated maximum speeds of tornado-borne missiles are given In Table 8, based on a set of assumptions believed to be reasonable. However, in assessing these speeds, it must be recognized that:

1. The problem of determining tornado-borne missile speeds has a probabilistic character. As shown in the body of the report, unfavorable initial conditions may obtain--to which there correspond relatively low probabilities of occurrence--for which the maximum missile speeds would be higher than in Table 8 . The estimation of such probabilities is beyond the scope of the investigation covered by this report.

2. Estimates of tornado-borne missile speeds are also affected by significant uncertainties with regard to: (a) the detailed structure of the tornado flow and (b) to the aeordynamic behavior of the missile. Under certain assumptions regarding one or both of these factors, calculated missile speeds can be higher than those of Table 8. However, it is believed that the assumption used to derive the values of Table 8 are conservative. In particular, it is believed that the actual vertical wind speeds are lower than indicated by $\mathrm{Eq} .9$, so that the relatively heavy missiles would tend to hit the ground sooner than calculated on the basis of this equation, with a consequent reduction in the calculated maximum missile speed.

In spite of the many uncertainties involved, the writers believe that the assumptions used to estimate the speeds of Table 8 are sufficiently conservative for purposes of nuclear power plant design. It is the writers' judgement that, although higher values of tornado-borne missile speeds are conceivable, their probabilities of occurrence, for any given tornado strike, are low. 
1. Paddleford, D.F., Characteristics of Tornado Generated Missiles, Report WCAP - 7897, Westinghouse Electric Corp. Pittsburgh,Pennsylvania, April, 1969.

2. Lee, A.J.H., Design Parameters for Tornado Generated Missiles, Topical Report GAI-TR-102, Gilbert Assoc., Inc., Reading, Pennsylvania, Jan., 1975.

3. The Generation of Missiles by Tornadoes, TVA-TR74-1, Tennessee Valley Authority, Nov., 1974.

4. Iotti, R.C., Velocities of Tornado-Generated Missiles, ETR-1003, Ebasco Services Inc., New York, N.Y., Feb., 1975.

5. Beeth, D.R., and Hobbs, S.H. Jr., Analysis of Tornado Generated Missiles, B\&R -001 , Brown \& Root, Inc., Houston, Texas, May 1975.

6. Meyers, B.L., and Morrow, W.M., Tornado Missile Risk Mode1, BC-TOP-10, Bechtel Power Corporation, San Francisco, Calif., June 1975.

7. Battacharya, A.K., Boritz, R.C., Niyogi, P.K., Characteristics of Tornado Generated Missile UEC-TR-002-0, United Engineers \& Constructors, Inc., Ph1ladelphia, Oct., 1975.

8. Fung, Y.C., An Introduction to the Theory of Aeroelasticity. Dover Publications, Inc., 1969.

9. Scanlan, R.H., "An Examination of Aerodynamic Response Theories and Model Testing Relative to Suspension Bridges," Proceedings, Third International Conference on Wind Effects on Bufldings and Structures, Tokyo, 1971.

10. Eagleman, J.R., Muirhead, V.U., Williams, N., Thunderstorms, Tornadoes and Building Damage, Lexington Books, Lexington, Mass., $197 \overline{5}$

11. Markee, E.H., Beckerley, J.G., Sanders, K.E., Technical Basis for Interim Reglonal Tornado Criteria, Wash-1300 (UC-11), U.S. Atomic Energy. Commission, Office of Regulation, May, 1974.

12. Bates, F.C., and Swanson, A.E., "Tornado Conslderations for Nuclear Power Plants," Transactions, American Nuclear Society, Vol. 10, pp. 712-713, Nov., 1967.

13. Hoecker, W.H., "Wind Speed and Air Flow in the Dallas Tornado of April 2, 1975," Monthly Weather Review, Vol. 88, No. 5, 1960.

14. Wortman, A., "Aerodynamics of Randomly Tumbling Bodies," Journal of Spacecraft and Rockets, AIAA, Feb., 1969, p. 205-207.

15. Hansche, G.E., and Rinehart, J.S., "Air Drag on Cubes At Mach Numbers 0.5 to 3.5," Journal of the Aeornautical Sciences, Feb., 1952, pp. 83-84.

16. Hoerner, S.F., Fluid-Dynamic Drag, Published by the Author, 1958.

17. National Design Specification for Stress-Grade Lumber and Its Fastenings, National Forest Products Association, Washington, D.C., 1973.

18. Garson, R.C., Catalan, J.M., and Cornell, C.A., "Tornado Design Winds Based on Risk," Journal of the Structural Division, ASCE, Vol. 101 No. ST9, Proc. Paper 11559, September, 1975, pp. 1883-1897.

19. McDonald, J.R., Mehta, K.C., Minor, J.E., "Tornado-Resistant Design of Nuclear PowerPlant Structures," Nuclear Safety,Vo1. 15, No. 4, July-August 1974. 
APPENDIX A - EQUATIONS OF MOTION

\section{Al.1 Absolute and Translating Frames of Reference}

Consider the absolute frame of reference $0_{1}$ xyz (Fig. Al.1) and a frame $0_{2} x^{\prime} y^{\prime} z$ such that $0_{2} z$ is parallel to $0_{1} z$. It is assumed that the frame $0_{2} x^{\prime} y^{\prime} z$ translates with respect to $0_{1}$ xyz with a constant velocity, the components of which (in the $0_{1}$ xyz frame) are $\left(v_{T x}, v_{T y}, 0\right)$. The angle between $0_{2} x^{\prime}$ and $0_{1} x$ being denoted by $\theta_{c}$,

$$
\begin{aligned}
& \cos \theta_{c}=\frac{v_{T x}}{\left(v_{T x}^{2}+v_{T y}^{2}\right)^{1 / 2}} \\
& \sin \theta_{c}=\frac{v_{T y}}{\left(v_{T x}^{2}+v_{T y}^{2}\right)^{1 / 2}}
\end{aligned}
$$

\section{A1.2 Vectors of Position}

Using the notations of Fig. Al.1, the vectors of position of the particle $P$ in the absolute and in the translating frame of reference may be written as follows:

Absolute frame (with origin $0_{1}$ )

$$
\bar{R}=x \bar{I}+y \bar{j}+z \bar{k}=r \cos \theta \bar{i}+r \sin \theta \bar{j}+z \bar{k}
$$

where $\overline{1}, \bar{\jmath}, \bar{k}$ are unit vectors along the axes $0_{1} x, 0_{1} y, 0_{1} z$ respectively.

Translating frame (with origin $\mathrm{O}_{2}$ )

$$
\bar{R}^{\prime}=x^{\prime} \bar{I}^{\prime}+y^{\prime} \bar{J}^{\prime}+z \bar{k}=r^{\prime} \cos \theta^{\prime} \bar{I}^{\prime}+r^{\prime} \sin \theta^{\prime} \bar{J}^{\prime}+z \bar{k}(\mathrm{Al} .4)
$$

where $\bar{I}^{\prime}, \bar{j}^{\prime}, \bar{k}$ are unit vectors along the axes $0_{2} x^{\prime}, 0_{2} y^{\prime}, 0_{2} z$, respectively

\section{A1.3 Transformations of Unit Vectors}

The unit vectors $\bar{r}, \bar{\theta}, \bar{k}$ of the revolving frame of reference (see Fig. Al.1) may 


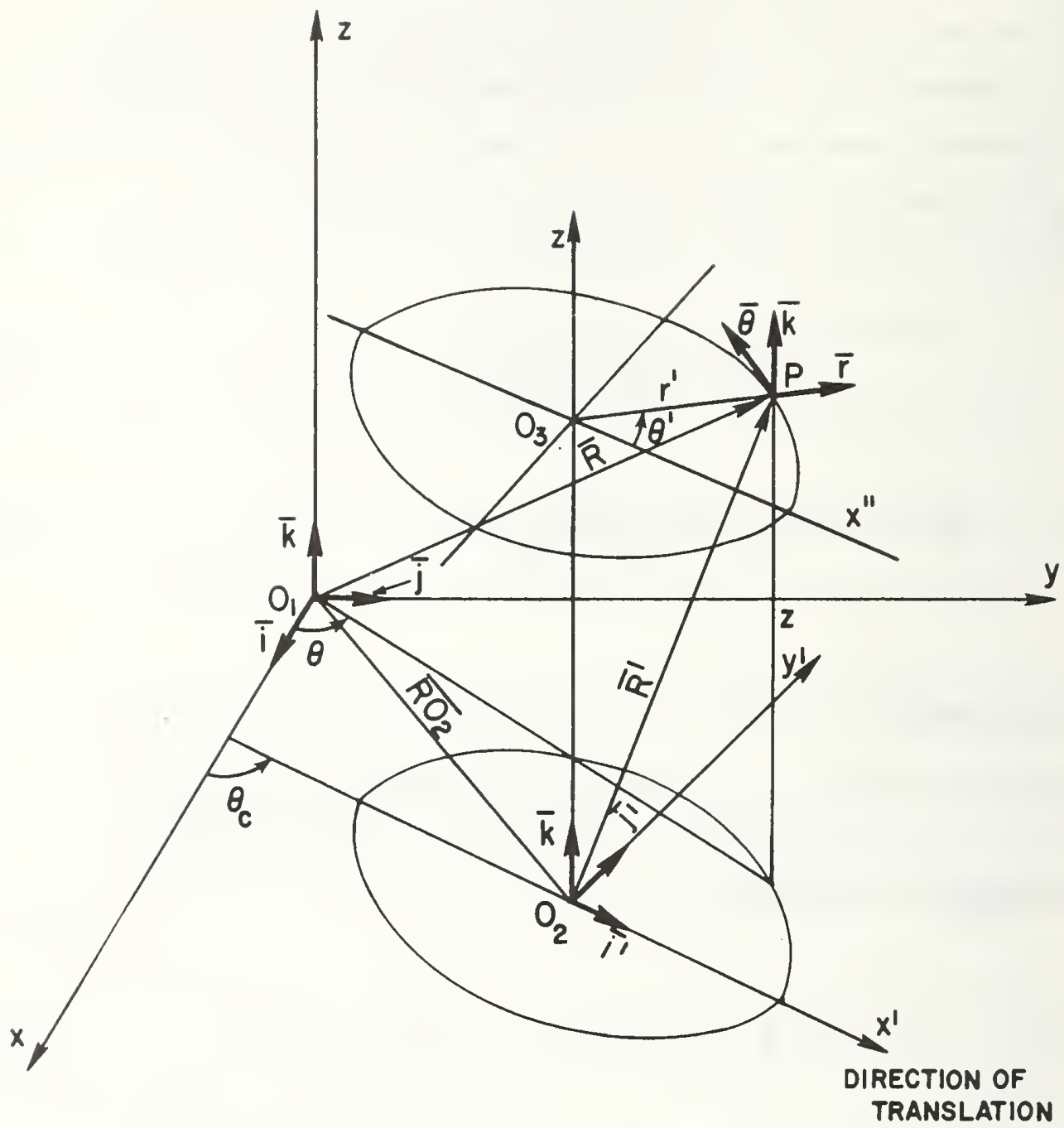

Figure Al Notations 
be written in terms of the unit vectors of the translating frame $0_{2} x^{\prime} y^{\prime} z$ and the angle between the lines $O_{3} P$ and $O_{3} x^{\prime \prime}$, where $0_{3} x^{\prime \prime}$ is parallel to $0_{2} x^{\prime}$, as follows:

$$
\begin{aligned}
& \bar{r}=\cos \theta^{\prime} \overline{1}^{\prime}+\sin \theta^{\prime} \bar{\jmath}^{\prime} \\
& \bar{\theta}=-\sin \theta^{\prime} \overline{1}^{\prime}+\cos \theta^{\prime} \bar{\jmath}^{\prime} \\
& \bar{k}= \\
& \bar{k}
\end{aligned}
$$

The unit vectors $\bar{I}^{\prime}, \bar{J}^{\prime}, \bar{k}$, of the translating frame $0_{2} x^{\prime} y^{\prime} z$ may be written in terms of the unit vectors of the absolute frame of reference and of the angle $\theta_{c}$ as follows:

$$
\begin{aligned}
& \bar{I}^{\prime}=\cos \theta_{c} \bar{I}+\sin \theta_{c} \bar{j} \\
& \bar{j}^{\prime}=-\sin \theta_{c} \bar{I}+\cos \theta_{c} \bar{\jmath} \\
& \bar{k}= \\
& \bar{k}
\end{aligned}
$$

\section{Al.4 Expression of Wind Velocity in an Absolute Frame of Reference}

Let the vortex wind velocity with respect to the translating frame of reference be denoted $\bar{v}_{\omega}$, and let 1ts components along the vectors $\bar{r}, \bar{\theta}$ and $\bar{k}$ be denoted by $v_{\omega r} \cdot v_{\omega \theta}$ and $v_{\omega k}$, respectively, 1.e.,

$$
\bar{v}_{\omega}=v_{\omega r} \bar{r}+v_{\omega \theta} \bar{\theta}+v_{\omega k} \bar{k}
$$

With respect to the absolute frame of reference, $\bar{v}_{\omega}$ may be written as

$$
\bar{v}_{\omega}=v_{\omega x} \bar{I}+v_{\omega y} \bar{\jmath}+v_{\omega z} \bar{k}
$$

The components $v_{\omega x}, v_{\omega y}$ and $v_{\omega z}$ are obtained by substituring Eqs. Al.5 and Al.6 into Eq. Al.7a, i.e., in matrix form, 


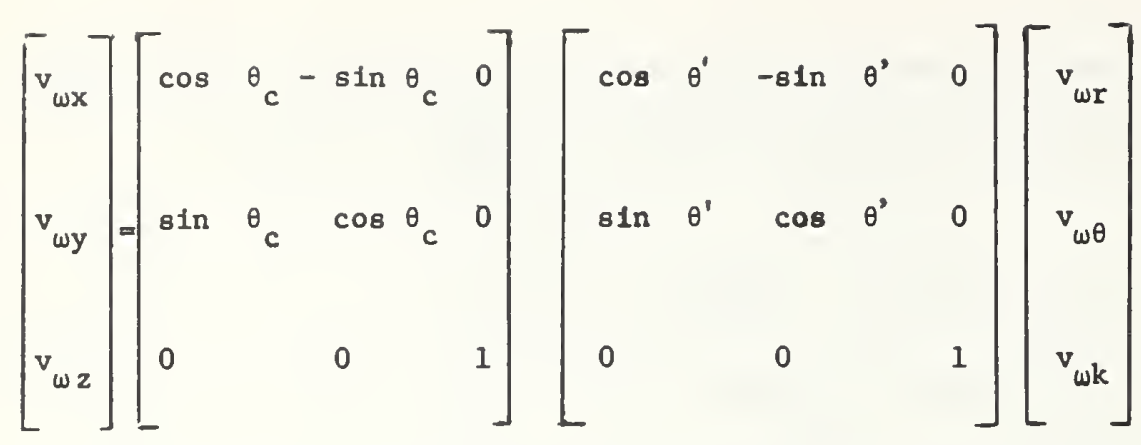

(Al.8)

The total wind velocity $\bar{v}_{w}$ consists of the sum of the vortet wind velocity and the translating velocity of the vortex, i.e.,

$$
\bar{v}_{w}=v_{w x} \bar{i}+v_{w y} \bar{j}+v_{w k} \bar{k}
$$

where

$$
\left.\begin{array}{l}
v_{w x}=v_{w x}+v_{T x} \\
v_{w y}=v_{w y}+v_{T y} \\
v_{w z}=v_{w z}
\end{array}\right\}
$$

and $v_{\omega x}, v_{\omega y}, v_{\omega z}$ are given by Eqs. A1.8. In Eqs. Al.10 and Al.8, the quantities $v_{T x}, v_{T y}, v_{\omega r}, v_{\omega \theta}, v_{\omega k}$ and $\theta_{c}$ are specifled. The quantity $\theta^{\prime}$ is a function of time and is obtained from the relations

$$
\left.\begin{array}{l}
\cos \theta^{\prime}=\frac{x^{\prime}}{r^{\prime}} \\
\sin \theta^{\prime}=\frac{y^{\prime}}{r^{\prime}} \\
r^{\prime}=\left(x^{\prime 2}+y^{\prime 2}\right)^{1 / 2}
\end{array}\right\}
$$


in which $x^{\prime}$ and $y^{\prime}$ are determined as explained in the following.

Let the initial conditions of the problem be

$$
\begin{aligned}
& t_{0} \equiv \text { inftial time } \\
& \left(x_{0}, y_{0}, z_{0}\right) \equiv \text { coordinates of particle at time } t_{0} \text { in the absolute frame } \\
& \left(v_{\mathrm{Pxo}}, \mathrm{v}_{\mathrm{Pyo}}, \mathrm{v}_{\mathrm{PzO}}\right) \equiv \text { velocity components of particle at time } t_{0} \text { in the } \\
& \text { absolute frame } \\
& \left(\mathrm{x}_{20}, \mathrm{yO}_{20}, 0\right) \equiv \text { position of } \mathrm{O}_{2} \text { (origin of the translating frame) at time } \\
& t_{0} \text {, in the absolute frame. }
\end{aligned}
$$

At time $t$, the position vector of the origin $0_{2}$ is

$$
\overline{\mathrm{RO}}_{2}=\left[\mathrm{xO}_{20}+\mathrm{v}_{\mathrm{Tx}}\left(t-t_{0}\right)\right] \bar{I}+\left[\mathrm{yO}_{20}+v_{\mathrm{Ty}}\left(t-t_{0}\right) \bar{\jmath}\right]
$$

so that for any time $t \geq t_{0}$ we have

$$
\overline{\mathrm{R}}^{\prime}=\overline{\mathrm{R}}-\overline{\mathrm{RO}}_{2}
$$

where

$$
\bar{R}=x \bar{I}+y \bar{j}+z \bar{k}, \text { I.e., }
$$

$$
\left[\begin{array}{c}
x_{R^{\prime}} \\
y_{R^{\prime}} \\
z_{R^{\prime}}
\end{array}\right]=\left[\begin{array}{l}
x \\
y \\
z
\end{array}\right]-\left[\begin{array}{l}
{ }^{x}{ }_{20}+v_{T x}\left(t-t_{0}\right) \\
{ }^{y} 0_{20}+v_{T y}\left(t-t_{0}\right) \\
0
\end{array}\right]
$$

Since

$$
x_{R^{\prime}} \bar{I}+y_{R^{\prime}} \bar{\jmath}+z_{R^{\prime}} \bar{k}=x^{\prime} \bar{I}^{\prime}+y^{\prime} \bar{\jmath}^{\prime}+z^{\prime} \bar{k}
$$

it follows, using the inverse of the transformation Al.6,

$$
\left[\begin{array}{l}
x^{\prime} \\
y^{\prime} \\
z^{\prime}
\end{array}\right]=\left[\begin{array}{ccc}
\cos \theta_{c} & \sin \theta_{c} & 0 \\
-\sin \theta_{c} & \cos \theta_{c} & 0 \\
0 & 0 & 1
\end{array}\right]\left[\begin{array}{c}
x_{R^{\prime}} \\
y_{R^{\prime}} \\
z_{R^{\prime}}
\end{array}\right]
$$


where $x_{R^{\prime}}, y_{R^{\prime}}, z_{R^{\prime}}$, are given by Eq. Al.14. The quant1t1es $x^{\prime}, y^{\prime}, z^{\prime}$, in Eq. Al.11, and thus the quantity $\theta^{\prime}$ In Eqs Al.10. Al.8 are expressed in terms of the coordinates of point P with respect to the absolute frame, and of the quantities $\mathrm{xO}_{\mathrm{xO}}, \mathrm{y}_{20^{\circ}}, \mathrm{v}_{\mathrm{Tx}}, \mathrm{v}_{\mathrm{Ty}}, \mathrm{t}_{\mathrm{o}}$ and $t$. The problem of expressing the components in the absolute frame of the wind velocity at point $P(x, y, z)$ in terms of the vortex wind velocity components $v_{\omega r}, v_{\omega \theta}, v_{\omega k}$, of the translation velocity components $v_{T x}, v_{T y}$ of the Initial conditions and of the coordinates $x, y, z$ of the point $P$ is thus solved.

\section{A1.5 Equat Ions of Motion}

The equations of motion may be written as

$$
m\left[\begin{array}{c}
\ddot{x} \\
\ddot{y} \\
\ddot{z}
\end{array}\right]=1 / 2 \rho C_{D} A\left|v_{\operatorname{Rel}}\right| \bar{v}_{\operatorname{Rel}}-8 \overline{\mathrm{k}}
$$

where $m=$ mass of particle, $0=$ alr density, $C_{D}=$ drag coefficient, $A=$ area of the particle, $\bar{v}_{\operatorname{Re} 1}=\left(v_{w x}-\dot{x}\right) \bar{I}+\left(v_{w y}-\dot{y}\right) \vec{J}+\left(v_{w z}-\dot{z}\right) \vec{k}_{1}$ and $g=$ acceleration of gravity.

APPENDIX B - ANALYTIC SOLUTION TO THE EQUATIONS OF MOTION FOR A UNIFORM WIND FIELD.

In the case of a unfform wind field an analytic solution of the equations of motion of a particle may be obtained as shown herein. This solution was used to test the computer program, in which the wind fleld subroutine was suitably modifled. The following assumptions were used :

1) The inftial velocity of the particle 18 zero

2) The motion occurs in the $x-z$ plane only

3) The wind velocity vector is at all points parallel to the horizontal axis $0_{x}$ and has the constant magnitude $v_{w}$. 
The equation of motion in the horizontal direction is

$$
\frac{d x^{2}}{d t^{2}}=\alpha\left(v_{w}-\frac{d x}{d t}\right)^{2}
$$

where

$$
\begin{gathered}
\alpha=1 / 2 \quad \frac{\rho}{m} C_{D} A, \text { or } \\
\int \frac{d\left(\frac{d x}{d t}\right)}{\left(v_{w}-\frac{d x}{d t}\right)^{2}}=\int \alpha d t+C_{1}
\end{gathered}
$$

With the change of variables $u=\frac{d x}{d t}-v_{w}, d u=d \frac{d x}{d t}$, Eq A22 becomes

$$
\begin{aligned}
& \int \frac{d u}{u^{2}}=\alpha t+c_{1} \\
& -\frac{1}{\frac{d x}{d t}-v_{w}}=\alpha t+c_{1} \\
& \frac{d x}{d t}=v_{w}-\frac{1}{a t+c_{1}}
\end{aligned}
$$

Integrating Eq. A2.5, there follows

$$
x=v_{w} t-\int \frac{d t}{\alpha t+c_{1}}+c_{2}
$$

W1th the change of variables $\alpha t+c_{1}=u, \alpha d t=d u, d t=\frac{d u}{\alpha}, E q . A 2.6$ becomes

$$
\begin{aligned}
& x=v_{w} t-\frac{1}{\alpha} \int \frac{d u}{u}+c_{2} \\
& x=v_{w} t-\frac{1}{\alpha} \ln \left(\alpha t+c_{1}\right)+c_{2}
\end{aligned}
$$

The constants of integration are determined as follows:

At $t=0, x=x_{0}$ and $\frac{d x}{d t}=0$, i.e., 


$$
\begin{array}{ll}
x_{0}=-\frac{1}{a} \ln c_{1}+c_{2} & \text { (from Eq. A2.8) } \\
0=v_{w}-\frac{1}{c_{1}} & \text { (from Eq. A2.5) }
\end{array}
$$

Thus

$$
\begin{aligned}
& c_{1}=\frac{1}{v_{w}} \\
& c_{2}=x_{0}-\frac{1}{\alpha} \ln v_{w}
\end{aligned}
$$

and

$$
x=x_{0}+v_{w} t-\frac{1}{\alpha} \ln \left(\alpha v_{w} t+1\right)
$$




\section{APPENDIX C}

DOCUMENTATION,

AND

SAMPLE INPUT AND OUTPUT

OF COMPUTER PROGRAM 


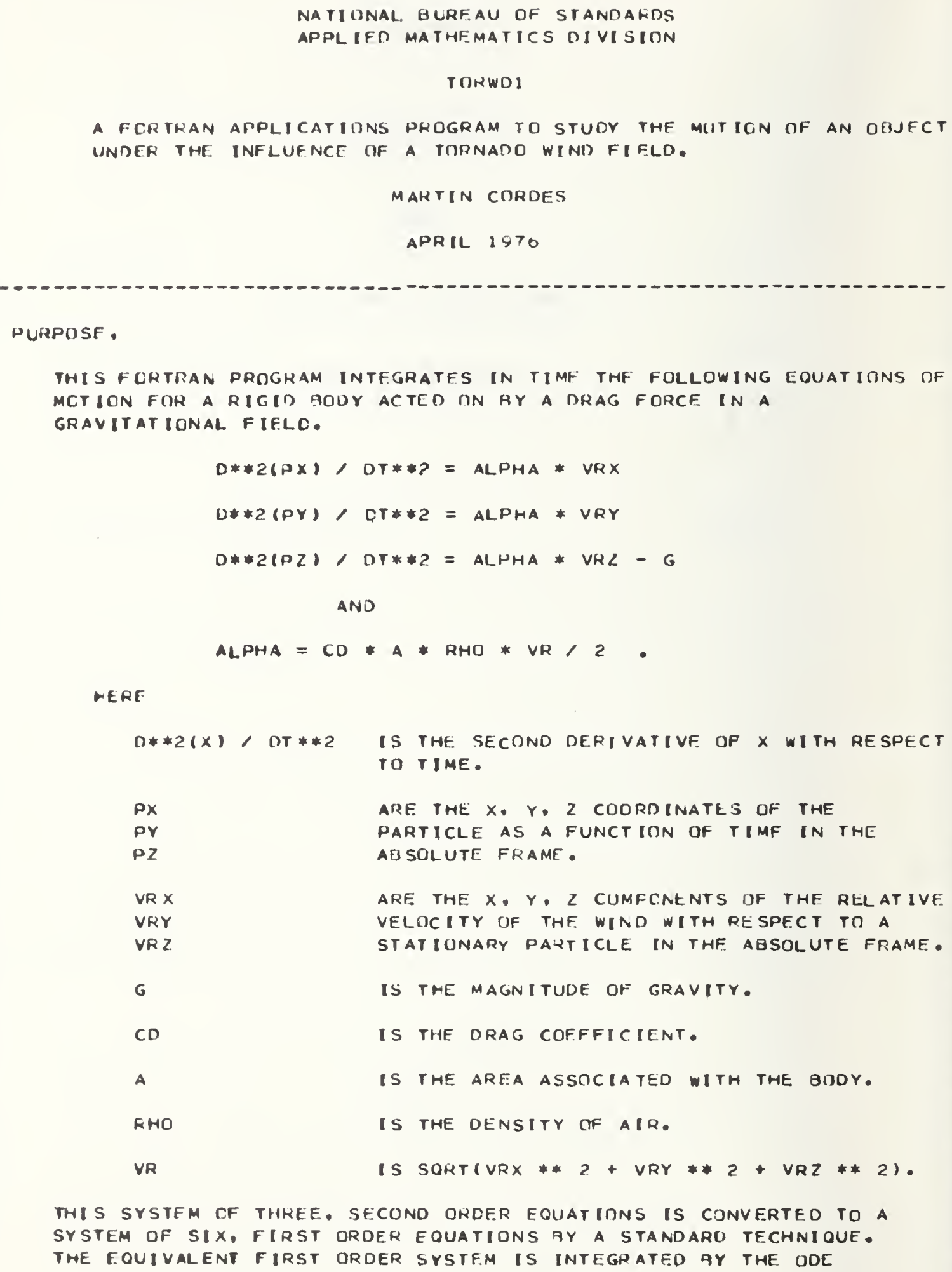


SOLVFR.

THE COMPUNENTS OF THE PARTICLE VELOCITY AND THE WIND VELTCITY APE WITH RESPECT TO AN ABSOLUTE FRAME. THF WIND VELDCITY IN THE ABSOLUTE FRANE IS THE SUM OF THE WIND VELDCITY FROM A STATIONARY TORNADO VORTEX AND THE TRANSLATICN VELOCITY OF THE TORNADC VOFTFX.

INITIAL CONDITIONS.

AT TINE = TO THE $X$. $Y$. $Z$ COORDINATES AND VELOCITY CGMPONENTS OF THE PARTICLE ARF SPECIFIED. IN ADDITICN. THE $X, Y$ COORDINATES OF THE ORIGIN OF THE TRANSLATING FRAME WHICH THE TURNADO IS STATIONARY IN ARE SPECIFIED.

CONDITIUNS INDEPENDENT OF TIME.

THE $X, Y$ VFLOCITY COMPONEATS OF THE TRANSLATING FRAME ARE CONSTANT.

FOR FURTHER DETAILS CONSULT REFERENCE 2 ).

APPLICAEILITY ANC RFSTRICTICNS.

THIS PROGRAM IS INTENUED FOR WORK THAT REOUIRES MODERATE ACCURACY AND MODERATE TIME PERIDOS OF INTEGRATION.

THF CURRENT VERSION PRODUCES PLOTS OF AT MOST 101 DATA POINTS. THE DATA IS FROM THE TABULAR.RFSULTS GENFRATED AT THE FIRST 101 PRINT STEPS. TO PREVENT A TRUNCATED PLOT FROM AEIAG GENERATED RE SURE THAT TIMEIT AND FXTMIT SATISFY TIMEIT ILT. 100 * FXTMIT. HEPE TIMEIT IS THE TIME INTERVAL OF INTEGRATION AND FXTMIT IS THE TIME INTFRVAL BFTWEEN SUCCESSIVE PRINT STEPS.

PROGRAM STRUCTURE.

SURPROGRAM DIRECTORY.

COMMENT ADDITIONAL SURPRDGRAMS OTHER THAN FORTRAN INSTFINSIC FUNCTIONS AND FORTRAN AASIC FXTERNAL FUNCTIONS NECESSARY FUR THE EXFCUTION CF THIS MAIN PROGRAM ARE LISTED BELU IN IN ALPHABETICAL ORDER. EACH IS ACCOMPANIED OY A SHURT FUNCTIONAL DESCRIPTION.

DFUN THIS SUERQUTINE COMPUTES THE RIGHT HAND SIDE OF THE FIRST ORDFR CDE SYSTEM. DY IS THE LOCAL NAME OF DFUN IN VOADAM.

DRAG (USER SUPPLIED.) THIS SUEROUTINE COMPUTES THF DRAG COEFFICIENT.

INPUT THIS SUAROUTINE READS IN OR CONTROLS THE READING IN OF ALL RELEVANT DATA AND PARAMETERS EXCEPT THF PARAMETERS REQUIRED BY THE ODE SOLVER AND WHICH AFFECT OUTPUT.

OUTPUT THIS SUBROUTINE READS IN PARAMETERS THAT CONTROL WHICH PLOTS ARE TO BE PRODUCED AND SUPFRVISES THE PRODUCTION AF ALL OUTPUT. 


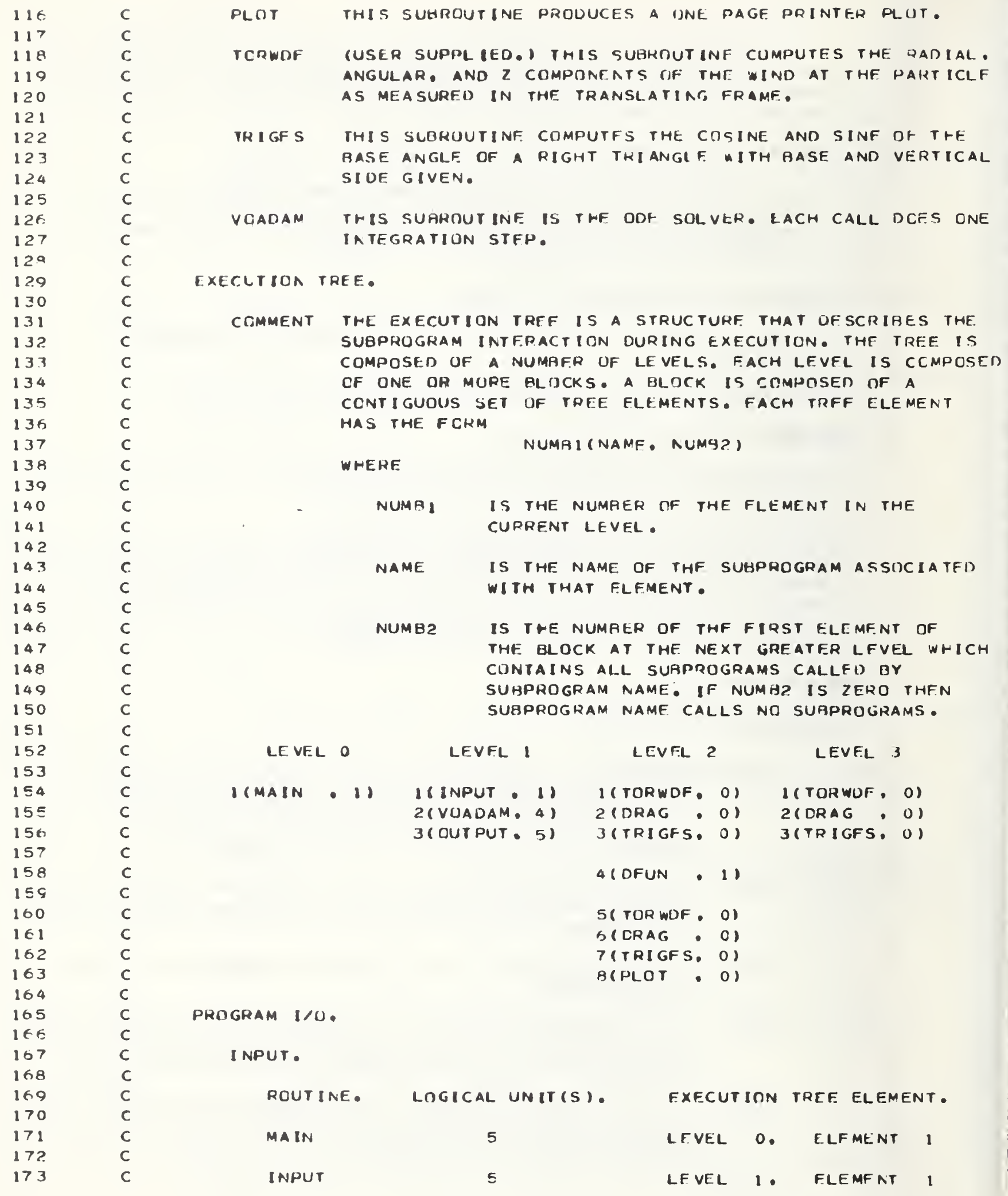


174

175

176

177

178

170

180

181

182

183

18.4

185

186

187

1 \&

$1 \mathrm{RS}$

190

191

152

193

194

195

196

197

198

199

200

201

202

203

204

205

206

207

208

209

210

2 11

212

213

214

215

216

217

218

2 19

<20

221

222

223

224

225

226

227

228

229

230

231 c

c

$\begin{array}{lccllll}\text { OUTPUT } & 5 & \text { LEVEL } & 1 . & \text { FLEMFNT } & 3 \\ \text { TORWOF } & 5 & \text { LEVLL } & 2 & \text { FLEMFNT } & 1 \\ \text { DRAG } & 5 & \text { LEVEL } & 2 . & \text { ELEMFNT } & 2\end{array}$




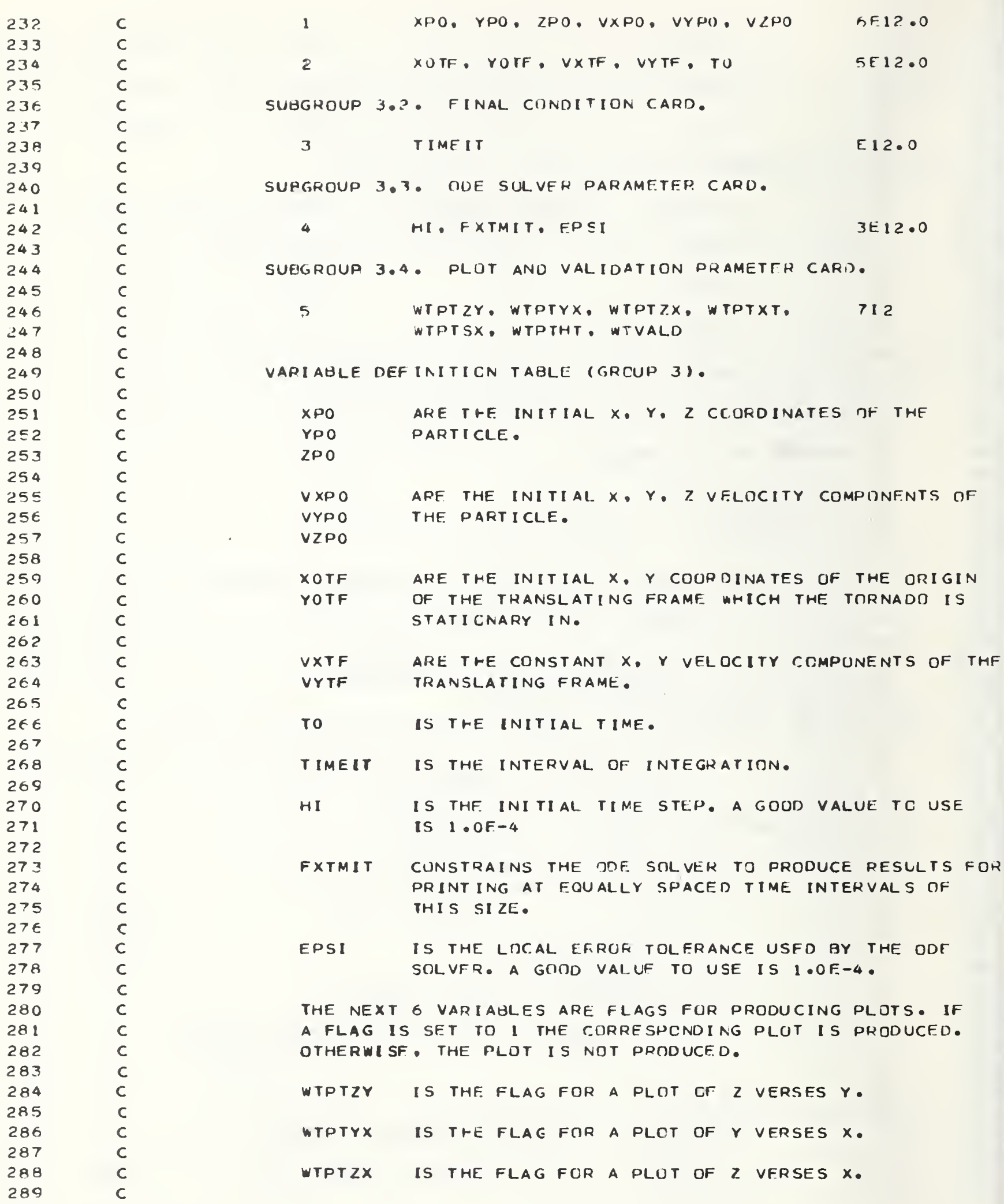

C
1
XPO. YPO, ZPO, VXPO. VYPO, VZPO
SF $12 \cdot 0$
2
XUTF. YOTF, VXTF, VYTF, TU
$5 E 12 \cdot 0$

SUBGHOUP 3.? FINAL CONDITION CARD.

3

TIME IT

E 12.0

SUPGROUP 3.3. OUE SULVER PARAMETEP CARD.

4

$$
\text { HI. FXTMIT, FPSI }
$$

उE $12 \cdot 0$

SUEGROUP 3.4. PLOT ANU VALIDATION PRAMETTH (ARI).

5

$$
\text { WTPTZY, WTPTYX, WTPTZX, WTPTXT. }
$$$$
712
$$$$
\text { WTPTSX, WTPTHT, WTVALD }
$$

VARI ABLE DEF INITICN TABLE (GRCUP 3 ).

$x P O$

ARE THE INITIAL $X, Y, Z$ CCORDINATES TF THE

YPO PART I CLE.

ZPO

$\checkmark \times P O$

$\checkmark Y P O$

ARE THE INITIAL $X, Y, Z$ VFLOCITY COMPIDNFNTS OF

$\checkmark Z P O$

THE. PART I CLE.

XOTF

YOTF

VXTF

VYTF

To

TIME IT

HI

IS THF. INITIAL TIME STEP. A GOOD VALUE TC USE IS $1 \cdot O F-4$

FXTMIT CUNSTRAINS THE DOE SOLVER TO PRODUCE RESULTS FOR PRINTING AT EOUALLY SPACED TIME INTERVALS OF THIS SIZE.

EPS I

IS THE LDCAL EFRUF TOLFRANCE USFD BY THE ODF SOLVFR. A GOND VALUF TO USE IS $1.0 F-4$.

THE NEXT 6 VARIABLES ARE fLAgS FUR PRODUCING PLOTS. If A FLAG IS SET TO I THE CORRESHCNDING PLUT IS PRODUCED. OTHERWISF. THE PLOT IS NOT PRODUCE.D.

WTPTZY IS THE. FLAG FOR A PLOT CF $Z$ VERSES $Y$.

WTPTYX IS THE FLAG fOR A PLOT OF $r$ VERSES $x$.

WTPTZX IS THE FLAG FOR A PLOT OF $z$ VFRSES $x$. 
C

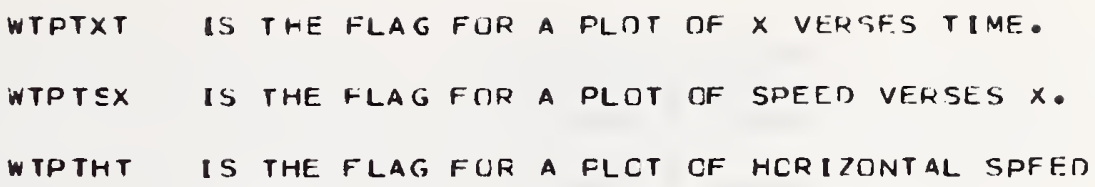




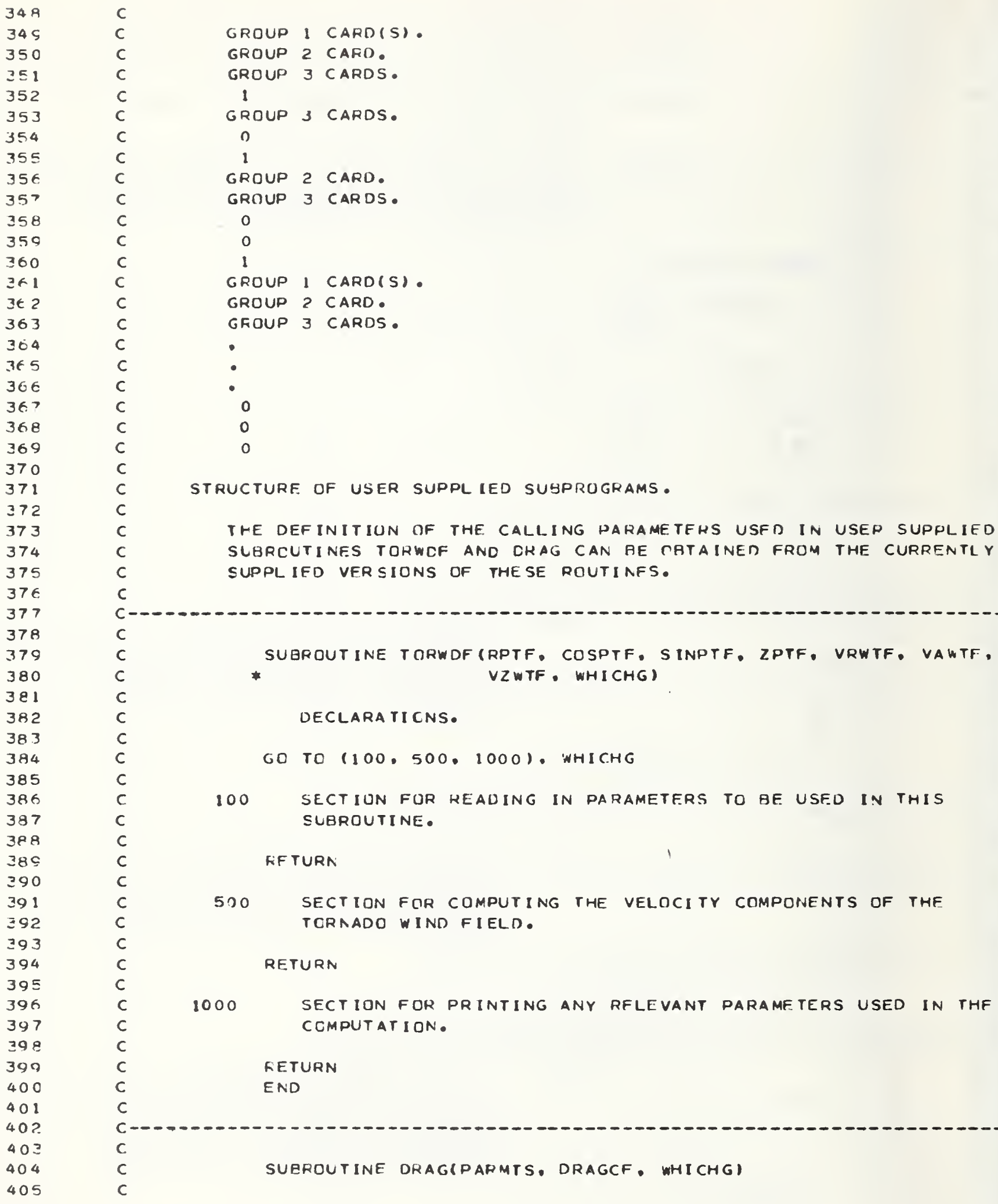

C 
DECLARAT I CNS.

GU TO $(100,500,1000)$, WHI CHG

100

SECTICN FCR READING IN FARAMFTERS TO BE USED IN THIS SUURCUT I NF.

RETURN

500 SECTION TOR CUMPUTING THF DRAG COEFFICIENT. RE TURN

SECTICN FOF PRINTING ANY RELFVANT PARAMETFRS USED IN THF COMPUTATION.

RE. TURN

END

\section{FRCGFAN USAGE (OUTPUT).}

LAYCUT OF OUTPUT.

THE PROGRAM OUTPUT IS BROKFN INTO 4 SECTICNS.

SECTICN 1. FROBLEM DESCRIPTION.

THE FCLLOWING DESCRIPT IVE INFORMATION IS PRINTED.

WIND VELDCITY PARAMETERS.

ALl THE PARAMETFRs WHICH ARE INPUTED For COMPUTED THE TOKNADO WIND FIELD ARE. PRINTED. THE NUMBER, NAMES, AND MEANING OF THF PARAMETERS ARE DETFRMINED BY THE USER.

DRAG COEFFICIFNT PARAMETERS.

ALL THE PARAMETERS WHICH ARE INPUTED FOR COMPUTING THF. DRAG COEFFICIENT ARE PRINTFD. THE NUMBFR, NAMES, AND MEANING OF THE PARAMETFFS ARE DETERMINFD BY THE USFR. PART ICLE PARAMETERS.

SELF -F XPLANATORY. FROM INPUT .

INITIAL CCNDITIONS (TORNADO WIND FIELO).

SELF-EXPLANATURY • FROM INPUT •

INITIAL CUNDITIONS (PAFTICLE).

SELF-EXPLANATORY. FROM INHUT.

SECTION 2. TABULAR RESULTS.

FIRST, TRE INITIAL TIME AND THF TIME INTERVAL FOR OUTPUT ARF: 


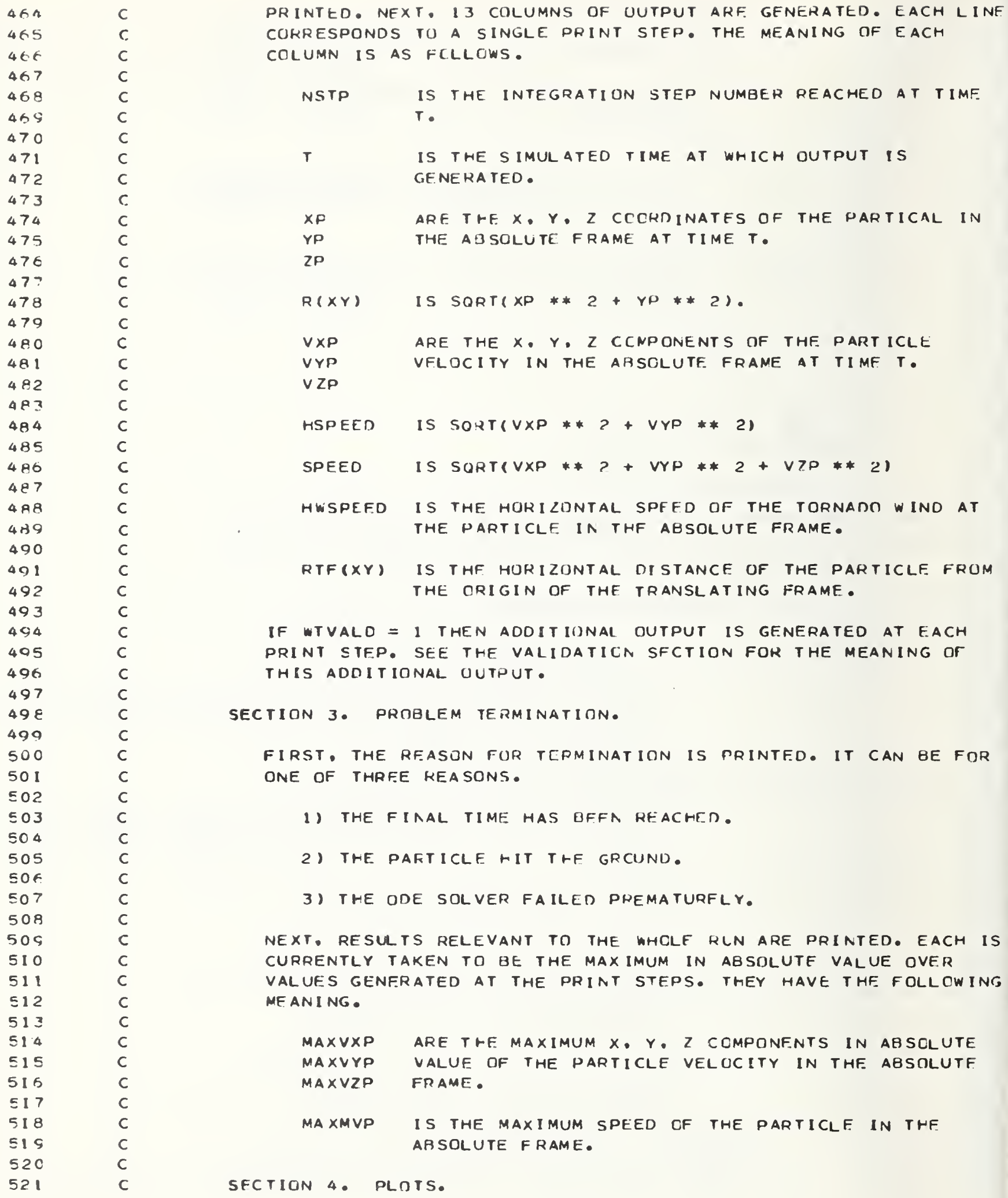

PRINTED. NEXT. 13 COLUMNS OF UUTPUT ARF. GENERATED. EACH LINF. CORRESPONDS TO A SINGLE PRINT STEP. THE MEANING OF EACH COLUMN IS AS FCLLOWS.

NSTP IS THE INTEGRATION STEP NUMBER REACHED AT TIMF $\mathrm{T}$.

$\mathbf{T}$

IS THE SIMUL ATED TIME AT WHICH OUTPUT IS GE.NERA TED.

$X P$

ARE THF $X$. $Y$. $Z$ CCCRDINATES OF THE PARTICAL IN

$Y P$

the agSOLUte frame at TIME T.

ZP

$R(X Y)$ IS SORT(XP**2+YP**2).

$\checkmark X P$ ARE THE $X$, $Y, Z$ CCNPONENTS OF THF PART ICLE

VYP VFLOCITY IN THE ABSOLUTE FRAME AT TIMF T.

$\checkmark Z P$

HSPEED IS SORT(VXP**?+VYP**2)

SPEED IS SORTIVXP * ? + VYP**2+VZP**21

HWSPEFD IS THE HORIZONTAL SPFED OF THE TORNADN WIND AT

THE PARTICLE IN THF ABSOLUTE FRAME.

RTF(XY) IS THF HORIZONTAL DISTANCE OF THE PARTICLF FROM THE ORIGIN OF THE TRANSLATING FRAME.

IF WTVALD = 1 THEN ADDITIONAL OUTPUT IS GENERATED AT EACH PRINT STEP. SEE THE VALIDATICN SFCTION FOR THE MEANING OF THIS ADDITIONAL UUTPUT.

SECTION 3. PROBLEM TERMINATION.

FIRST. THE RFASUN FUR TEPMINATION IS RRINTFD. IT CAN BE FOR ONE OF THRFE REASONS.

1) THE final time has bFFn REAChED.

2) THE PAFTICLE HIT THF GRCUNU.

3) The ode solver failed prematurfly.

NEXT. RESULTS RELEVANT TO THE WHCLF RLN ARE PRINTED. EACH IS CURRENTLY TAKEN TO BE THE MAXIMUM IN ABSOLUTE VALUE OVER VALUES GENFRATED AT THE PRINT STEPS. THEY HAVE THF FOLLOWING ME. AN I NG. 
LFRO OR MCRF PLOTS ARE PRODUCFN ONE PER PAGE. THF CHDINATE AND ABSCISSA LAHELS CAN BF FCUND CENTERED BFLOW THE GRAPH. THE PLOTTING CHARACTFR IS THE LFTTER $X$. AS NOTFO IN THF SFCTION. APPLICABILITY AND RFSTKICTIUNS. AT MOST IOI DATA POINTS ARE PLCTTED.

ERRCR HANOL ING.

INPUT CHECKING.

THE FOLLOWING VARIABLES ARE CHECKFO FOR VALIDITY IN THF. MAIN PROGRAM. IF AT LEAST ONE IS INVAL IO AN ERRDR MESSAGE IS PRINTED AND THFY ARF ALL RESFT TO DEFAULT VALUES. THE DFFAULT VALUES CAN BF FOUND IN THE SECTICN. MACHINE/SYSTFM DEPENDFNT FEATURES. AT THE START OF THE FXFCUTABLE CCOE IN THIS MAIN PROGRAN.

HI IS THE INITIAL STEP. IT MUST RE POSITIVE AND LESS THAN FXTMIT.

FXTMIT IS THE TIME INTERVAL USFD FOR OUTPUT. IT MUST BE PกSITIVE.

PROGRAM DETECTABLF ERRORS.

AFTER EACH INTEGRATICN STEP THE VARIABLE IFR IS CHECKED. IF ITS VALUE INDICATES THAT THE LAST STEP FAILED THEN INTEGRATION TERMINATES PREMATURELY. THE REST OF TMF. OUTPUT IS GFNERATED AS USLAL WITH THE ERRCR CODE PRINTED FRR THE REASUN. THE CUTPUT WILL REFLECT ONLY RESLLTS GENERATED UP TC THE TIME OF THE EFROR •

DISCUSSIUN OF METHOD AND ALGORITHM.

THE PROBLEM TO BE SOLVED IS A NUMERICAL INITIAL VALUE PRCRLEM IN ORDINARY DIFFERFNTIAL EQLATIONS. SIX, FIRST CRDEF, ORDINARY DIFFERENTIAL EQUATIONS, REPRESENTING THE EQLATIONS OF MOTION, ARE INTEGRATED FROM SOME INITIAL TIME WITH SPFCIFIED INITIAL CONDITIONS TO SOME LATER TIME WHICH SATISFIES SOME TERNIMATION CCNDITION.

THE ODE SQLVER, VIADAM, IS BASED ON THF VARIABLE ORDER, VARIABLE STEP ADANS CCCE DESIGNEC AND INPLEMENTATED EY C. W. GEAR IN RFFERENCE 1). SINCE THE SYSTEM DF NDES TO BE SOLVED IS NCN-STIFF ALL PARANETERS AND CALLS TO SUBROUTINES REQLIRED FOR SOLVING STIFF SYSTEMS HAVE BEFN DELETEN.

CN EACH CALL TC VOADAM THE ODE SOLVER IS ASKED TO INTEGRATE THE SYSTEM OF ODES OVER A STEP OF LFNGTH H. THE VALUE OF $H$ ARISES FROM CNE OF TWO SOURCES.

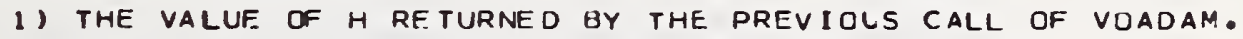

2) THE VALUE CF H SPECIFIED BY CALLER INTERACTION.

SOURCE 2) IS USED ONLY FOR THF INITIAL STEP AND WHEN THE STEP IS MODIFIED SC THAT IT FALLS IN A MULTIPLE OF THE FIXED TIME INTFRVAL USED FCR FRINTING. CTHFFWISE. SOURCE I) IS USEO SO AS TO 
C

C

C

c

c

C

C

C

C

C

C

C

C

C

C

C

C

C

C

C

C

C

C

C

C

C

C PORTABILITY. HMAX.

\section{VAL IUATICA.}

FCUSTF PSINTF

WRTF WANGTF WTF

WXTF WYTF

WXAF W YAF ZAF

THE INPUT VALUE. OF H IS USFO UNLESS THE FRRCR CRITERIA CANNOT BF MET. IN THIS CASE THE STEP AND/CR DFDFE ARE MOCIFIED TO TRY TO MEET THE FRROR CRITERIA. IF AN ATTENPT IS MADF IN REDUCE. THE STFP BFLOW A CALLER SUPP. IFD VALUE, MMIN, THE DDE SILVFR DUITS AND RETURNS THE APRROPRIATF NONZERO EFHCH CTNDITION CIIDE.

ONCF A SUCCESSFUL STEP HAS HEEN TAKEN, VDADAM ESTIMATFS AND RE:TURNS A GCOD VALUE OF M TO HE USED FOR THE NFXT STEP. THIS FSTIMATED STEP CANNCIT RE GREATFR THAN A CALLER SUFPLIED VALUF,

For MURE DETAILS UN VOAUAM CONSUL THE MACHINE REATABLE DOCUMENTATICN AT THE BEGINNING OF THF SUBROUTINE.

THE PROGRAM FRCVIDES THE USER BY WAY OF THE WTVALD INPUT VARIAGLE THE CAPABILITY OF PRINTING IMPOPTANT INTERMFDIATE OUANTITIFS. THESE OUANTITIES ARF PRINTED AS ADDIT IUNAL OUTPUT AT THE NORMAL PRINTING STEPS. THE AMOUNT OF OUTPUT PER PRINT STEP INCREASES FROM ONF LINE TO SEVEN LINES. TO INTFRPRET THE MEANING OF THE VARIABI.ES PRINTE, CONSULT THE TAGLE GFLOW aCCOMPANITD EY APPENDIX a of REFERENCE 21 .

RTF IS THE CYLINDRICAL RADIUS OF THF ORIGIN OF THE TRANSL. ATING FHAME FROM THF ORIGIN OF THE ABSCLUTF. FRAME.

CUStF ARE thF COSINE, SINE UF the ANGLE THAT the DIRECTION SINTF CF TRANSLATICN MAKES WITH THE $X$ aXIS OF THE ab SOLUTE FRAME •

PRTF IS THF CYLINDKICAL RADIUS OF THE PARTICLE FROM THF. ORIGIN OF THE TRANSLATING FRAME. are the cos Ine, Sine of the angle that the CYLINDRICAL RADIUS TO THE PARTICLE MAKES WITH THF $X$ AXIS OF THF. TRANSLATING FRAME. ARE THE RADIAL, angular, and $Z$ CCMPONENTS OF THE WIND VFLOCITY AT THE PARTICLE USING THF REVOLVING FRAME. ARE THE $X$. Y COMPONF, NTS OF THE IND VFLOCITY AT THE PARTICLE USING THE TRANSLATING FRAME. ARE THE $X, Y, Z$ COMPONENTS UF THE WIND VELOCITY AT THE PARTICLE USING THE ABSCLUTE FRAME.

RVXPAF RVYPAF PVZPAF ARE THE $X, Y, Z$ COMPONEATS OF THE RELATIVE VELOCITY OF THE PART ICLE WITH RESPECT TO THE WIND USING THE ABSOLUTE FRAME. 


$$
\begin{aligned}
& \text { c LANGUAGE. } \\
& \text { c } \\
& \text { c HELL VERIFIER FCRTRAN. }
\end{aligned}
$$

PRECISION.

SINGLE.

RESTHI CT IONS .

THIS PKUGRAM WAS DESIGNFO TO RUN CURRFCTLY WITH A MINIMUN CF MODIFICATION ON MACHINES WHICH HAVE A SINGLE PRECISION FLOATING POINT WORD WITH A MANTISSA IN THE RANGE OF 24 THROUGH 48 EITS.

ALL INPLT IS FROM FORTRAN FORMATTEC CARD IMAGES AND IS READ FROM LOGICAL UNIT 5 .

ALL OUTPUT IS FORTRAN FORMAT GENERATED AND IS WRITTEN TC LOGICAL UNIT 6. THE INTENCED OFVICE IS A LINE PRINTER SET TO AT LFAST A 132 CHARACTERS NER LINE ANC AT LEAST 60 LINES PER PAGE.

FUR FURTHER DETAILS SEF THE SFCTION, MACHINE/SYSTEM DFPENDENT FEATURES, AT THE BEGINNING OF THE EXECUTABLE CODF. IN THIS MAIN FRUGRAM AND ALL SUBPROGRAMS LISTFO IN THF SURPROGRAM DIRECTORY.

CCDE FESPCASIBILITY.

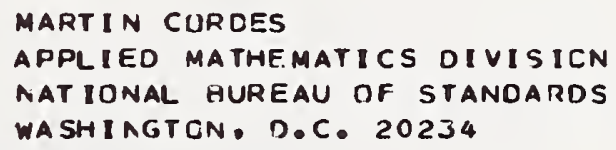

ORIGINAL VERSION.

NAY 1975

REVISEO VERSICA(S).

AUG 1975

APR 1976

REFERENCES •

1) C. W. GEAR. NUMERICAL INITIAL VALUF PFOBLEMS IN ORDIAAFY DIFFERENTIAL EQUATIONS. PRENTICF-HALL . 1971.253P.

2) E. SIMIU AND M. CORDES. TORNADO - BCRAE MISSILE SPEEDS. NBS INTERAGENCY REPORT 
SUBRCUTINF TCEWDF(FPTF, CCSPTF, SINPTF, LPTF, VRITH, VAWTF, VZWTF, * WH [ $\mathrm{CHG}$ ]

C

C

C

C

C

c

C

c

c

c

C

C

c

C

C

C

VRWTF, VAWTF, VZWTF

WH I CHG

PARAMFTER CFCLARATILNS.

REAL RPTF, COSPTF, S INPTF, ZPTF, VRWTF, VAWTF, VZWTF

INTEGEK WHICHG

C

C

c

C

C

c

c

C

c

MACHINE/SYSTEM CEPENUENT FEATURES.

DEFINITION OF I/O UNITS USED IN THIS SURROUTINE.

I 0

IS THE UNIT USFD FOR INPUTING DATA.

CD

IS TRE UNIT USED FDR CUTPUTING RFSULTS.

DATA ID. $00 / 5.6 /$

C

c

C

c

GO TO $(100,500,1000)$, WHICHG

C

C C

100 FEAC (IC.250) RMTVZO,MTV.K I.K2,K3,K4,K5

250 FORMAT(1CE\&.0) RFTURN TRANSLATIAG FRAMF TKANSLATING FRAME

KEAL RMTVZ,R33Z,RATIO

INTEGER ID, OD

READ IA IND VELCCITY PARAMETEFS

= RADIAL CCCRDINATE, COSINF. ANO SINE OF THE ANGLE. AND Z CCCROINATE OF THE DART ICLE WITH RESPECT TO THE C YLINDRICAL CCORDINATE SYSTFM DEFINED AS TRF

2 - COMPUTE WINO VFLOCITY COMPONENTS

3 - PHINT WIND VELOCITY PARAMFTERS 


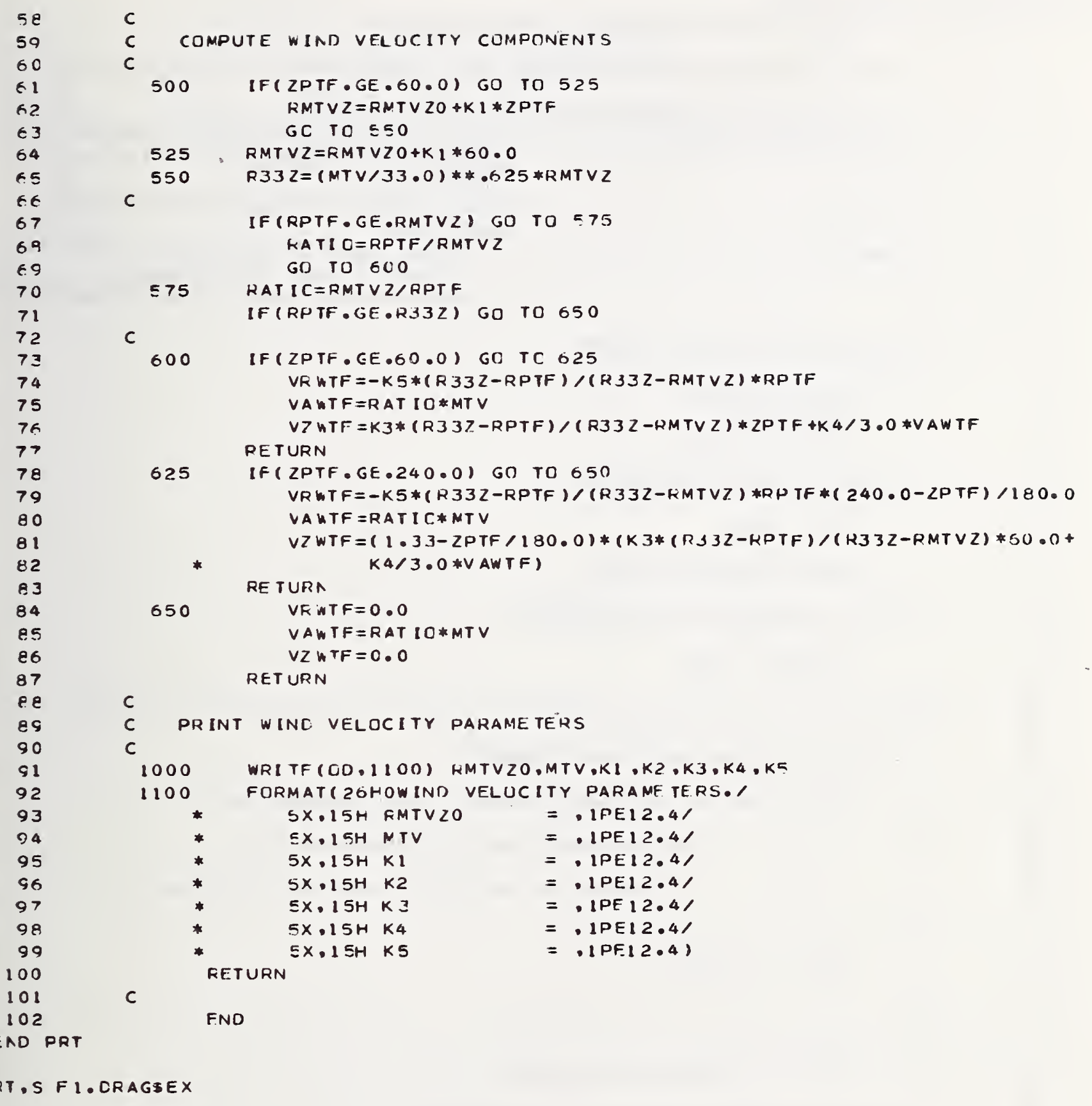


SURRUUTINF DRAG(PARMTS.URAGCF. NHICHG)

C

C this subroutine computes the dRAg coefficient for the makticle.

C

C DEF INITION OF PARAMETFRS. PARMTS(1)

- aRkAY OF TIME DCPENDENT PIRAME TE LS THAT AFFECT THE COMPUTATIUN OO THE DRAG COFFFICIFAT

URAGCF

= DRAG COEFFICIFNT COMPUTED RY THIS SURRUUTINF.

$W H I C H$

$=1$ - RFAT IN DFAT, COEFFICIFNT MARAMFTERS 2 - COMPUTF DRAG COLFFICIENT

3 - PRINT CIRAG COEFFICIENT FARAMFTIES

C PARAMETFR DECLARATICNS.

c

HEAL PARNTS(1)

RF.AL DRAGCF

INTEGFR WHICHG

C

C

OTHER COMMON VARIABLES.

REAL CURAG

C

CCMMCN / CGCOPA/CERAG

C

C

C

LCCAL VARIABLE CECLARATIIJNS.

INTFGER ID, OD

C

$\mathrm{C}$

$\mathrm{C}$

C

c

c

c

C

c

c

c

MACHINE/SYSTEM DEPENDENT FFATURES.

DEFIAITION OF I/C UNITS USFD IN THIS SUEROUTINE.

ID IS THE UNIT USED FOR INPUTING DATA.

$C D$

IS THE UNIT USFD FOR MLTPUTING FESULTS.

c

DATA IC, OD/5, 6/

c

$\mathrm{C}$

c

GC TO $(100,500,1000)$, WICHG

C

C READ IN DRAG COEFFICIFNT PARAMETFRS

C

100 READ(ID.250) CDRAG

250 FORMAT(6E.12.0)

RETURN

C

C CUMPUTE DRAG COEFFICICNT

c

$500 \quad$ DRA GCF $=$ CDRAG 


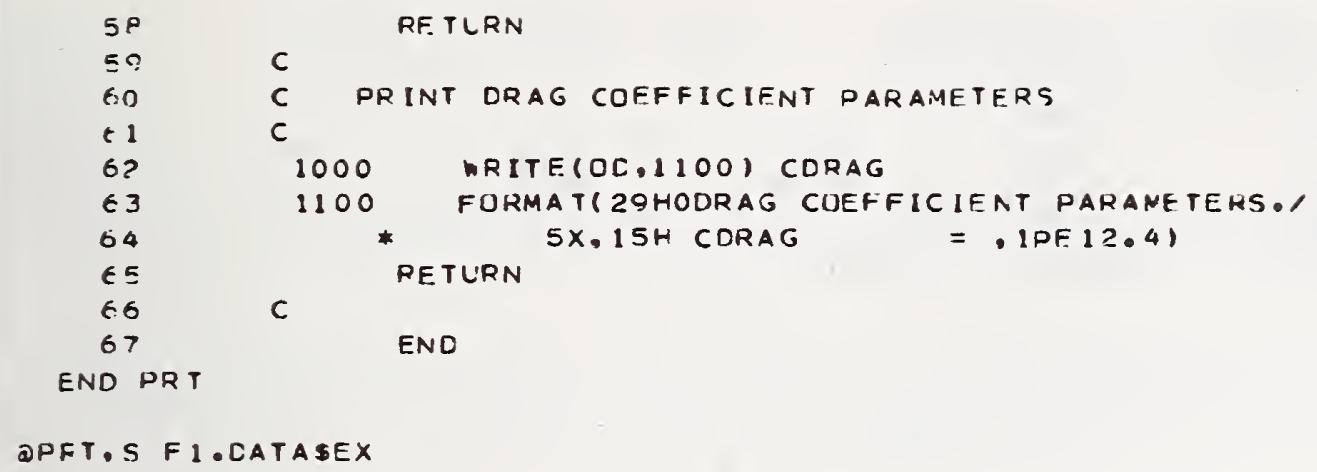


TCRAADO WIAD $(1)$. CATAStXX(1)

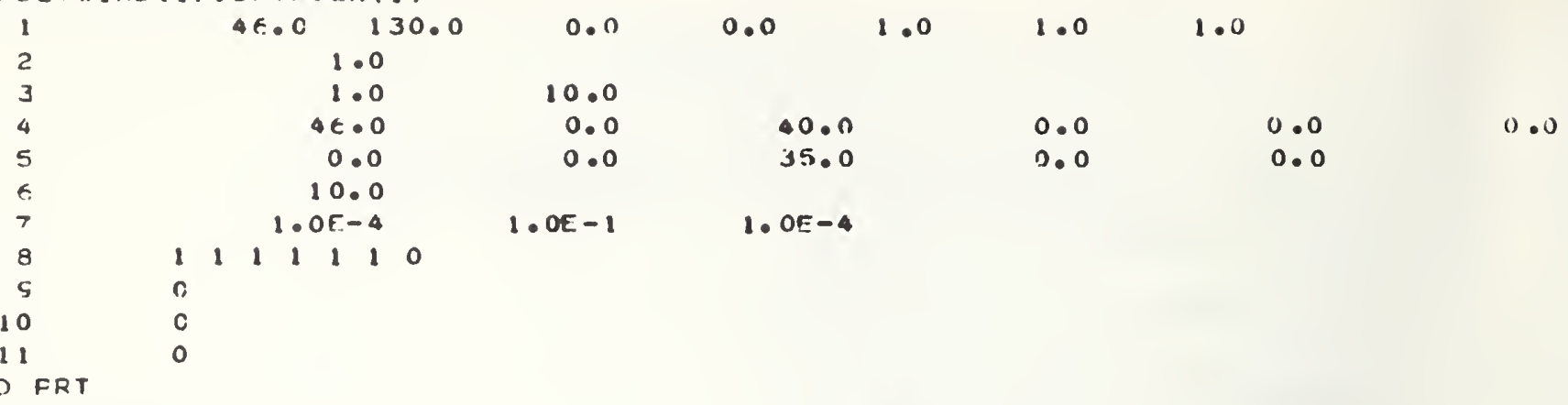

QXOT FI.ABSSEX 


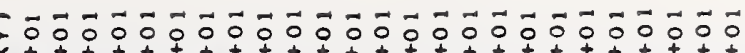

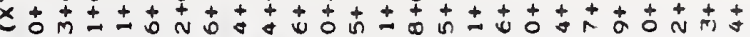

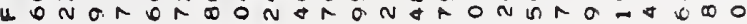

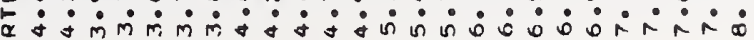

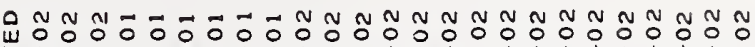

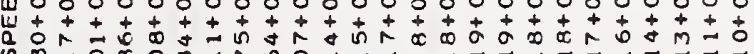

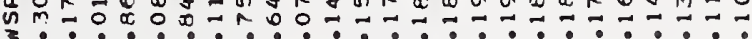

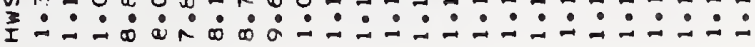

- -0 O

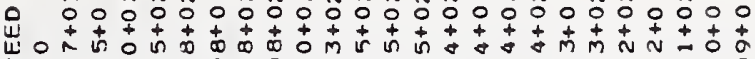

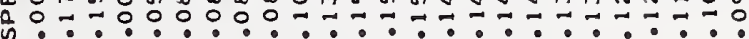

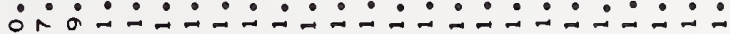

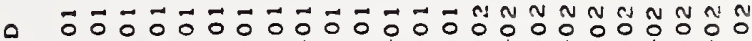

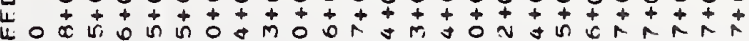

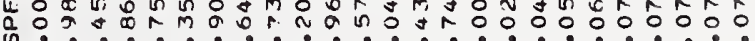

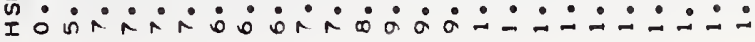

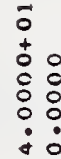

"II

$N \stackrel{N}{>}$

$z$
0
0
0
0
0
0
0
$z$
$z$
0
0
0
0
0
0

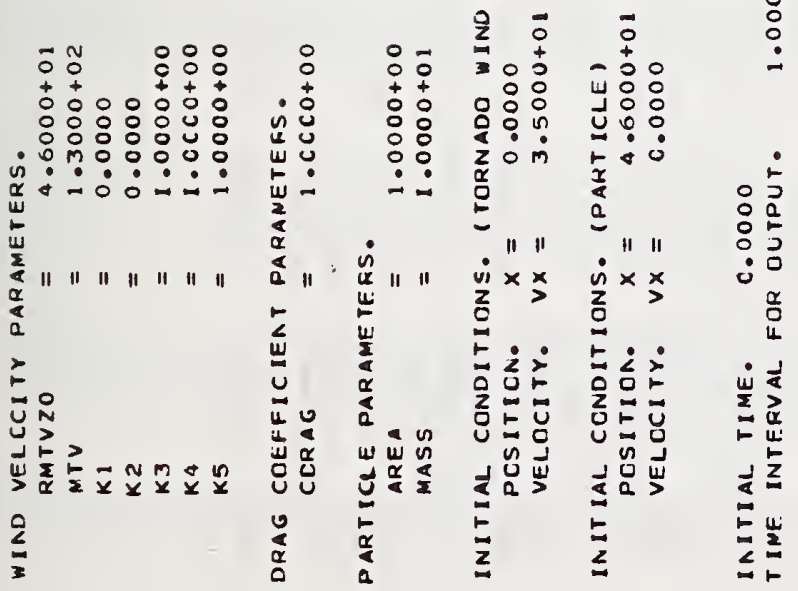

$\overrightarrow{0} \overrightarrow{0} \overrightarrow{0} \overrightarrow{0} \overrightarrow{0} \overrightarrow{0} \overrightarrow{0} \overrightarrow{0} \overrightarrow{0} \overrightarrow{0} \overrightarrow{0} \overrightarrow{0} \overrightarrow{0} \overrightarrow{0} \overrightarrow{0} \overrightarrow{0} \overrightarrow{0} \overrightarrow{0} \overrightarrow{0} \overrightarrow{0} \overrightarrow{0} \overrightarrow{0} \overrightarrow{0} \overrightarrow{0} \overrightarrow{0} \overrightarrow{0}$

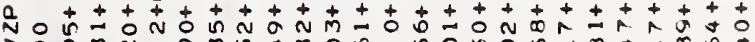

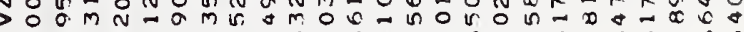

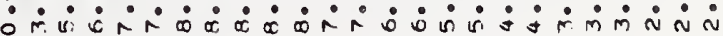

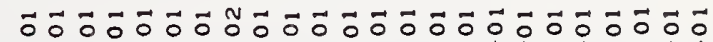

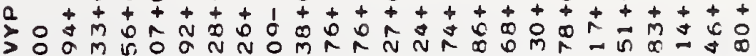

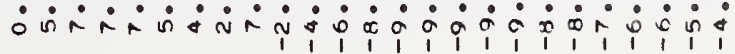

ㅁ $\overrightarrow{0} \overrightarrow{0} \overrightarrow{0} \overrightarrow{0} \overrightarrow{0} \overrightarrow{0} \overrightarrow{0} \overrightarrow{0} \overrightarrow{0} \overrightarrow{0} \overrightarrow{0} \overrightarrow{0} \overrightarrow{0} \overrightarrow{0} \overrightarrow{0} \overrightarrow{0} \overrightarrow{0} \overrightarrow{0} \overrightarrow{0} \overrightarrow{0} \overrightarrow{0} \overrightarrow{0}$

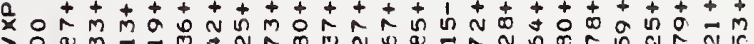

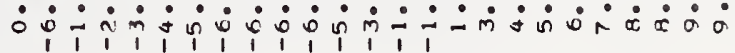

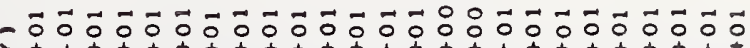

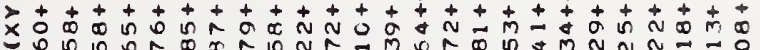

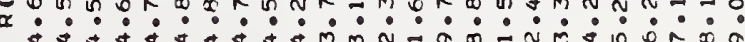

$\overrightarrow{0} \overrightarrow{0} \overrightarrow{0} \overrightarrow{0} \overrightarrow{0} \overrightarrow{0} \overrightarrow{0} \overrightarrow{0}$ ก

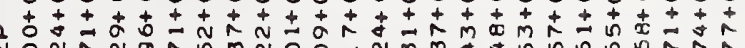

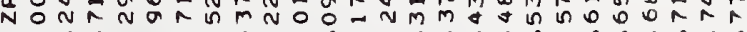

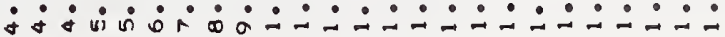

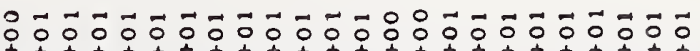

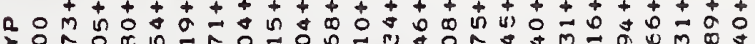

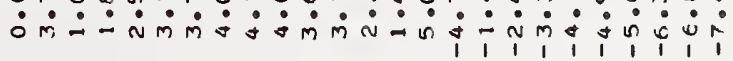

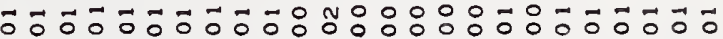

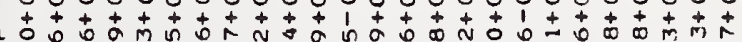
$x$ und

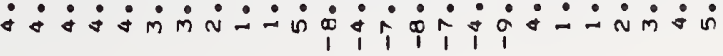

H H F H H

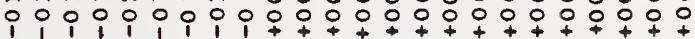

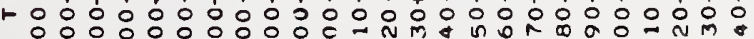

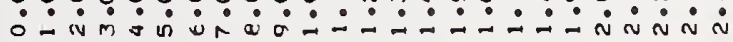

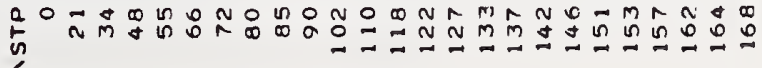




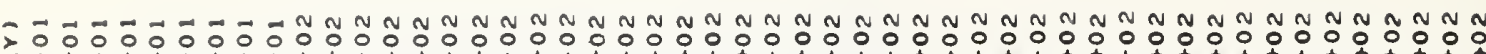

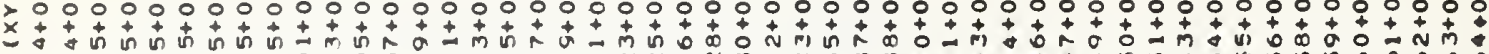

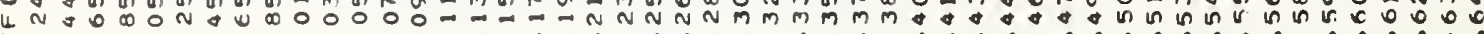
5

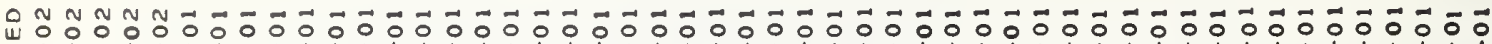

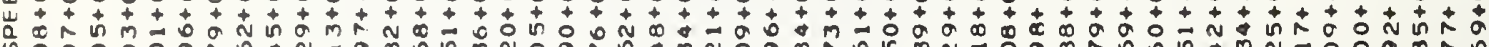

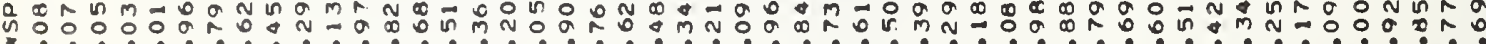

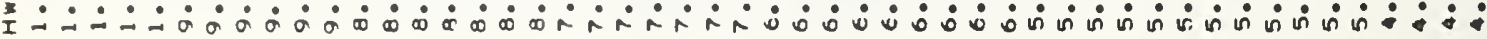

O

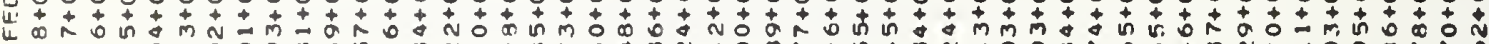

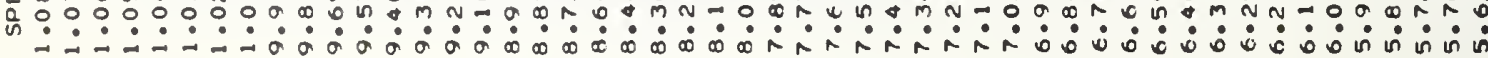

C

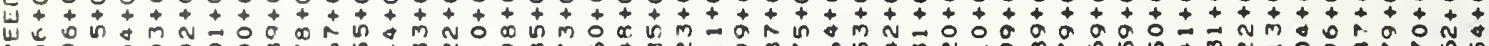

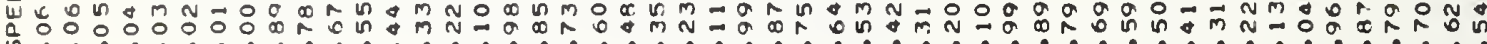

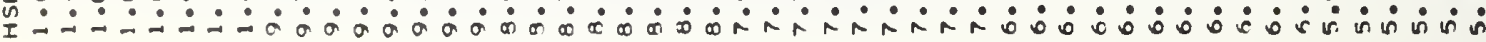

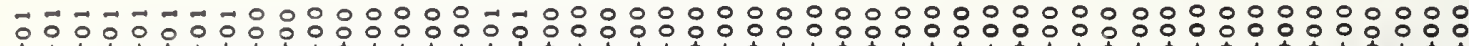

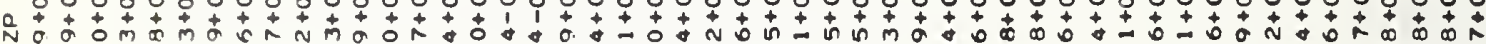

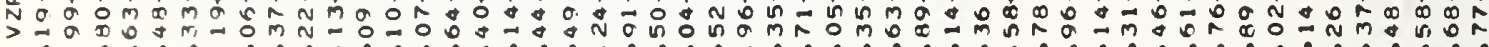

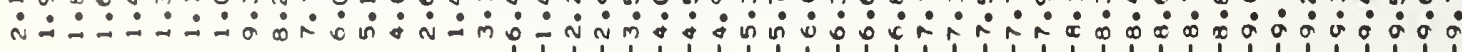

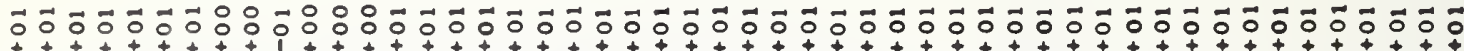

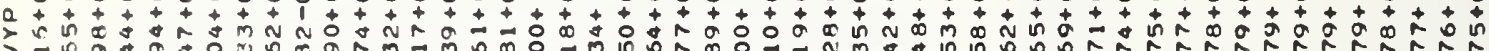

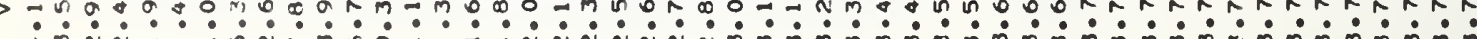

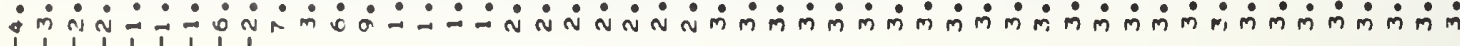

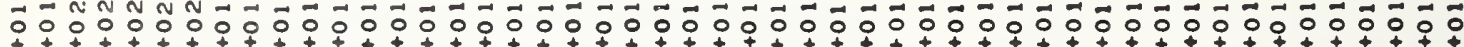

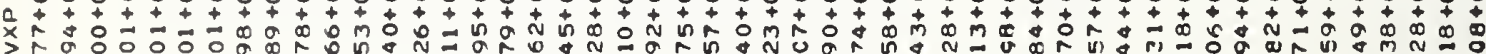

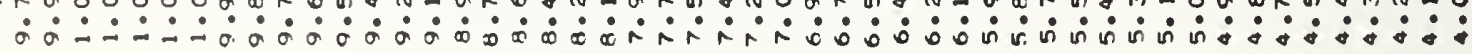

-

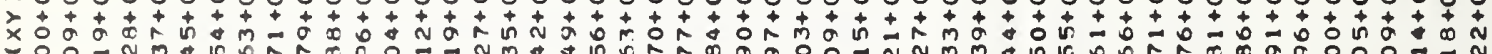
过:

N

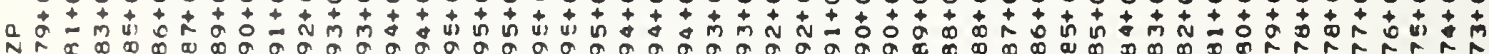

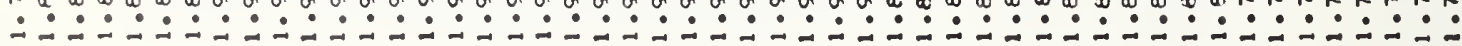

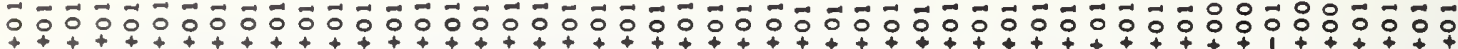

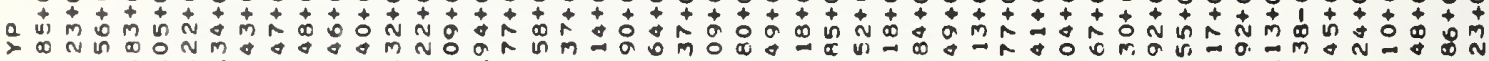

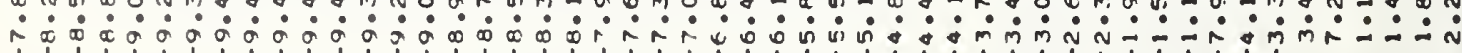

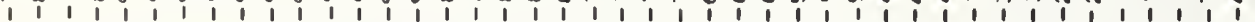

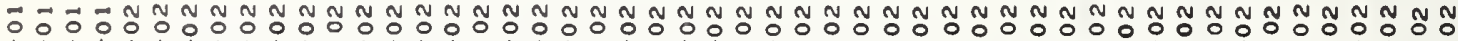

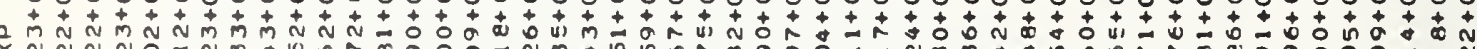

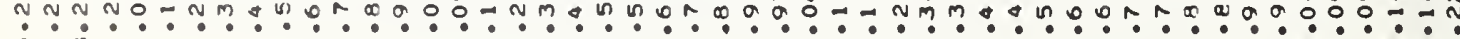

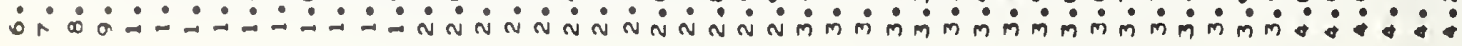

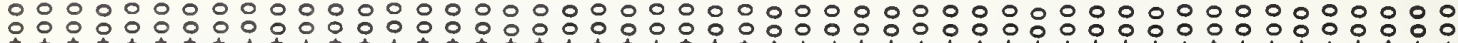

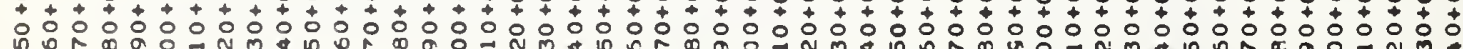

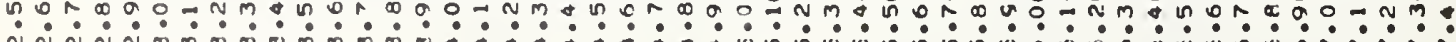

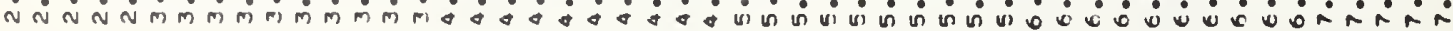

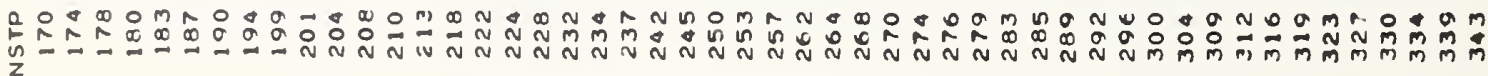




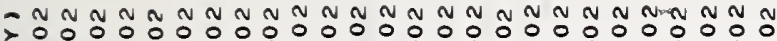

$x^{\prime}$
$>$

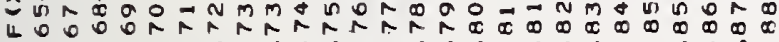

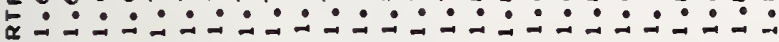

पी $\overrightarrow{0} \overrightarrow{0} \overrightarrow{0} \overrightarrow{0} \overrightarrow{0} \overrightarrow{0} \overrightarrow{0} \overrightarrow{0} \overrightarrow{0} \overrightarrow{0} \overrightarrow{0} \overrightarrow{0} \overrightarrow{0} \overrightarrow{0} \overrightarrow{0} \overrightarrow{0} \overrightarrow{0} \overrightarrow{0} \overrightarrow{0} \overrightarrow{0} \overrightarrow{0} \overrightarrow{0} \overrightarrow{0} \overrightarrow{0} \overrightarrow{0} \overrightarrow{0}$

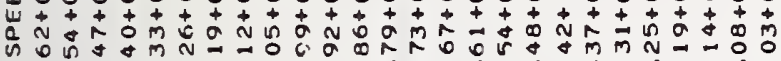

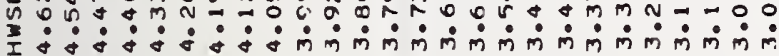

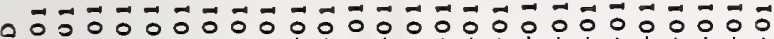

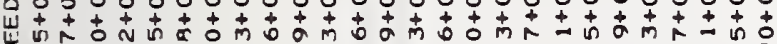

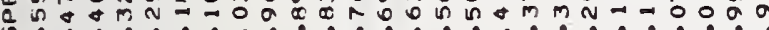

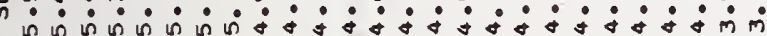

0 $\overrightarrow{0} \overrightarrow{0} \overrightarrow{0} \overrightarrow{0} \overrightarrow{0} \overrightarrow{0} \overrightarrow{0} \overrightarrow{0} \overrightarrow{0} \overrightarrow{0} \overrightarrow{0} \overrightarrow{0} \overrightarrow{0} \overrightarrow{0} \overrightarrow{0} \overrightarrow{0} \overrightarrow{0} \overrightarrow{0} \overrightarrow{0} \overrightarrow{0} \overrightarrow{0} \overrightarrow{0} \overrightarrow{0} \overrightarrow{0} \overrightarrow{0} \overrightarrow{0}$

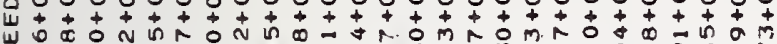

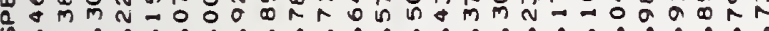

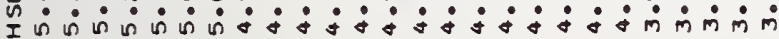

$\therefore: \overrightarrow{0} \overrightarrow{0} \overrightarrow{0} \overrightarrow{0} \overrightarrow{0} \overrightarrow{0} \overrightarrow{0} \overrightarrow{0} \overrightarrow{0} \overrightarrow{0} \overrightarrow{0} \overrightarrow{0} \overrightarrow{0} \overrightarrow{0} \overrightarrow{0} \overrightarrow{0} \overrightarrow{0} \overrightarrow{0} \overrightarrow{0} \overrightarrow{0} \overrightarrow{0} \overrightarrow{0} \overrightarrow{0} \overrightarrow{0}$

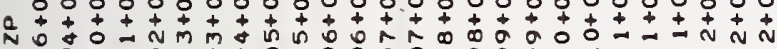
$>\infty 00000000000000000 \div \div \div \div \div-1$

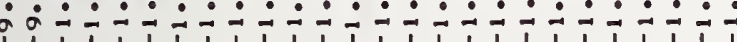

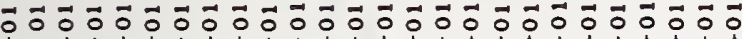

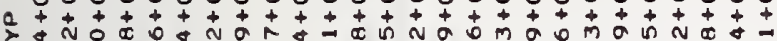

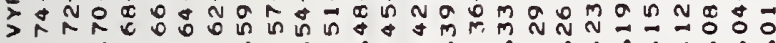
$\dot{m} \dot{m} \dot{m} \dot{m} \dot{m} \dot{m} \dot{m} \dot{m} \dot{m} \dot{m} \dot{m} \dot{m} \dot{m} \dot{m} \dot{m} \dot{m} \dot{m} \dot{m} \dot{m} \dot{m} \dot{m} \dot{m} \dot{m}$

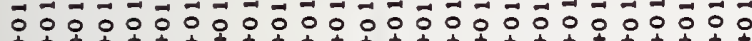

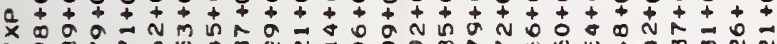
$\dot{m} \dot{m} \dot{m} \dot{m} \dot{m} \dot{m} \dot{m} \dot{m} \dot{m} \dot{m} \dot{\sim} \dot{N} \dot{N} \dot{N} \dot{N} \dot{N} \dot{N} \dot{N} \dot{N} \dot{N}$

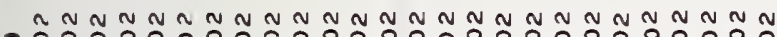

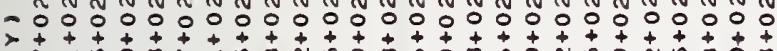

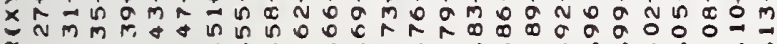

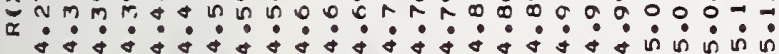

N

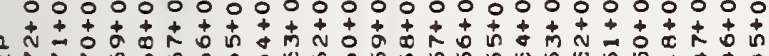

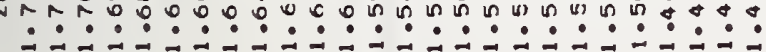

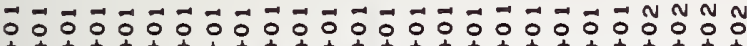

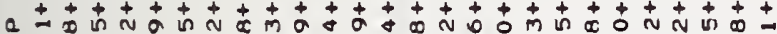
>

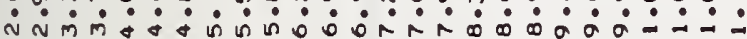

N

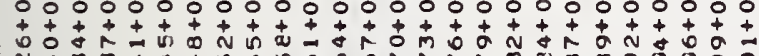

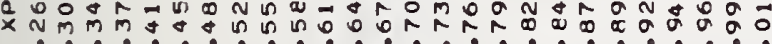
$\dot{+} \dot{0} \dot{0} \dot{0} \dot{0} \dot{0} \dot{0} \dot{0} \dot{0} \dot{0} \dot{0} \dot{0} \dot{0} \dot{0} \dot{0}$

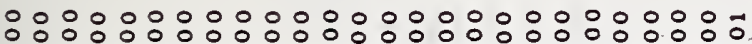

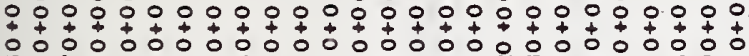

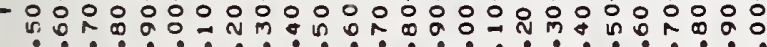

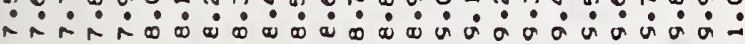

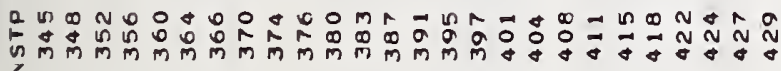




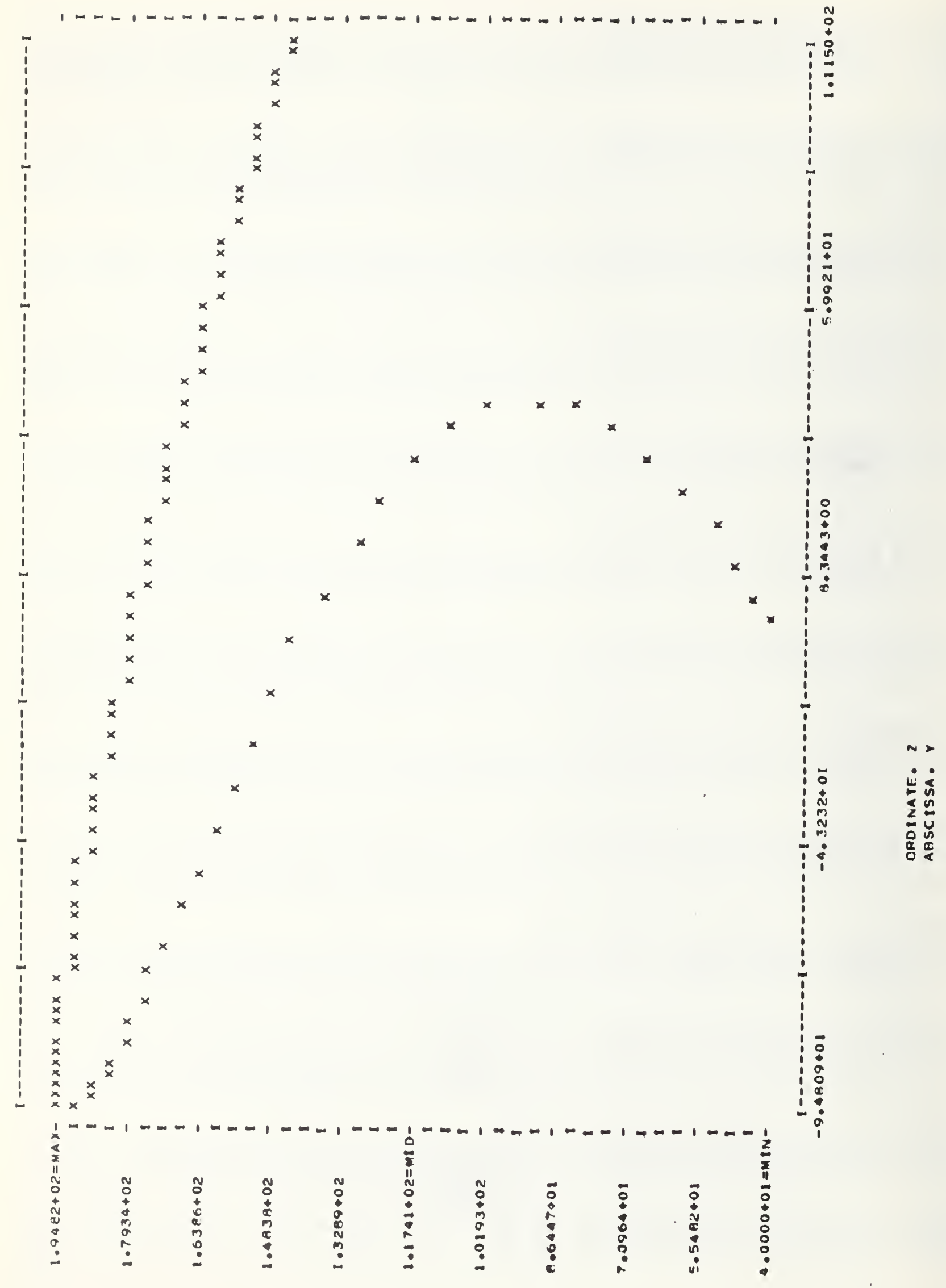




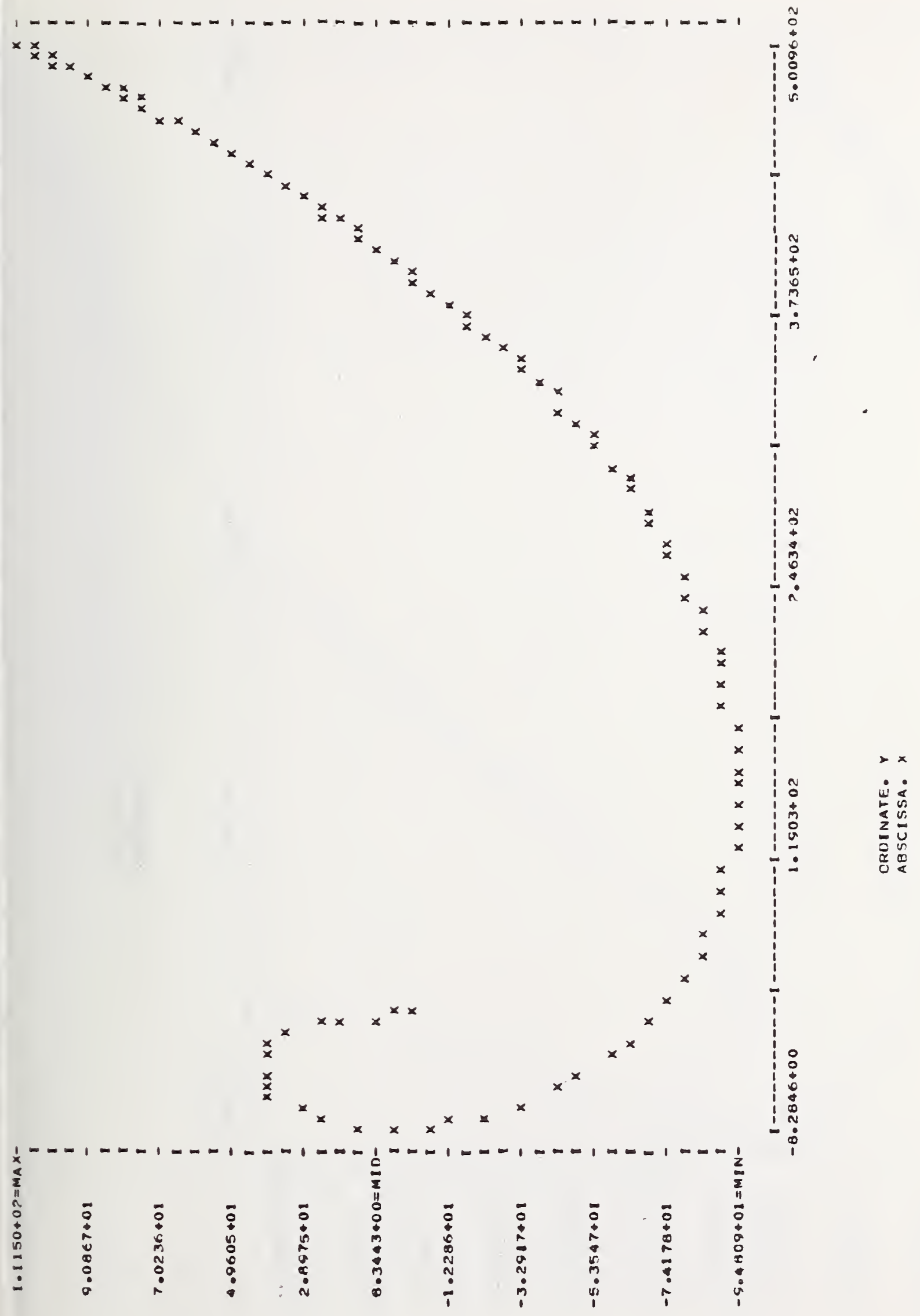




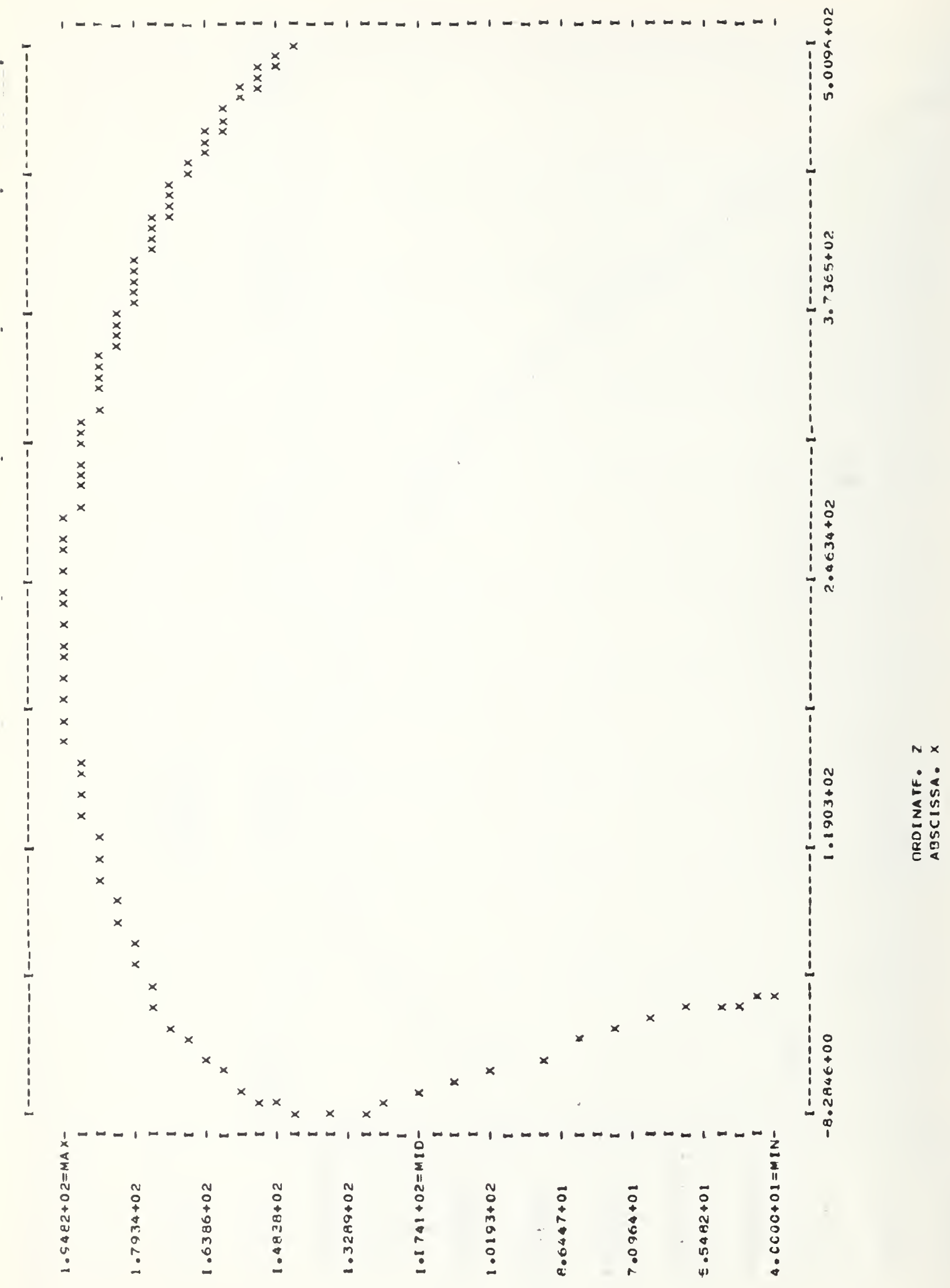




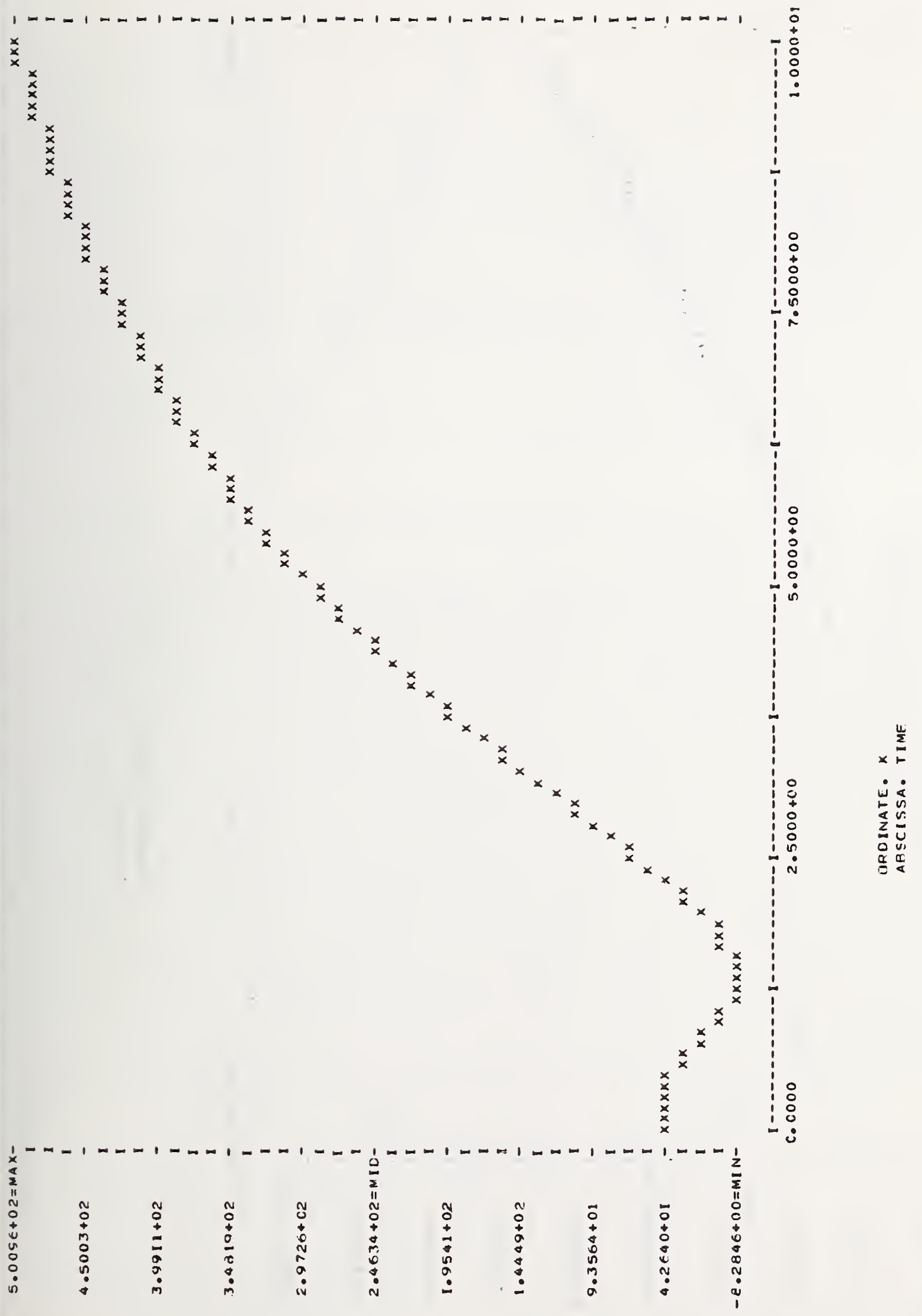




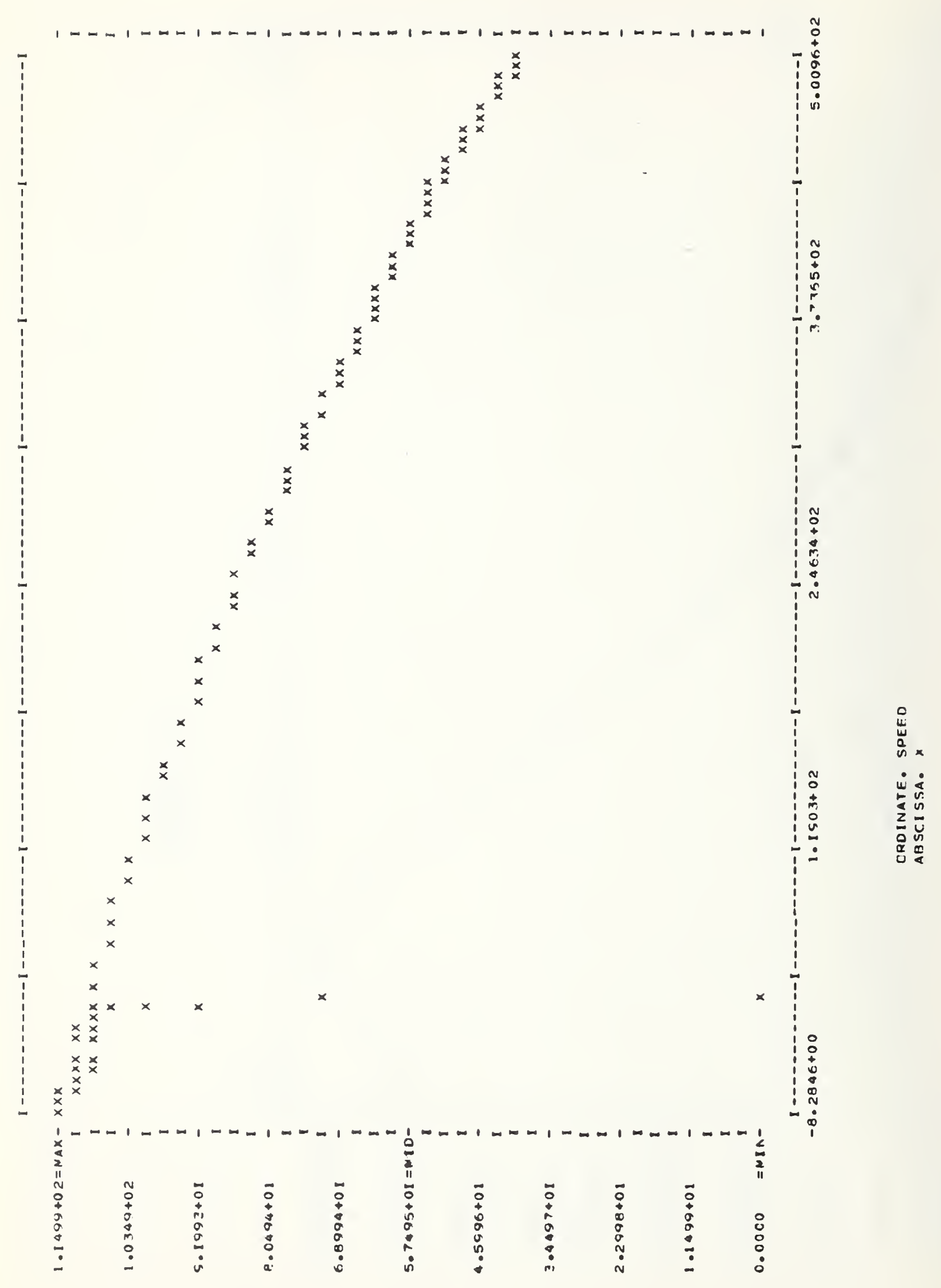




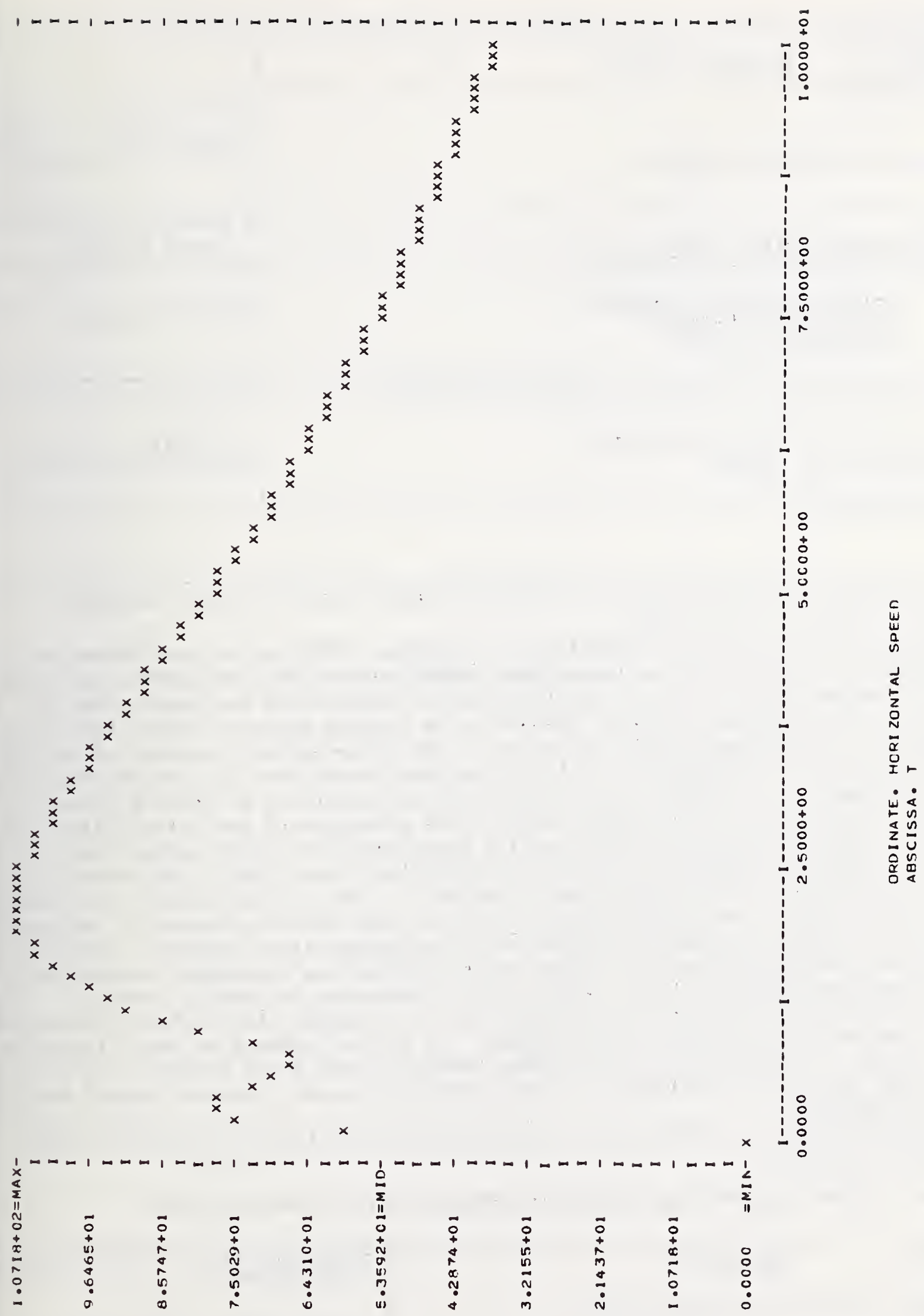


NBS-114A (REV. 7.73 )

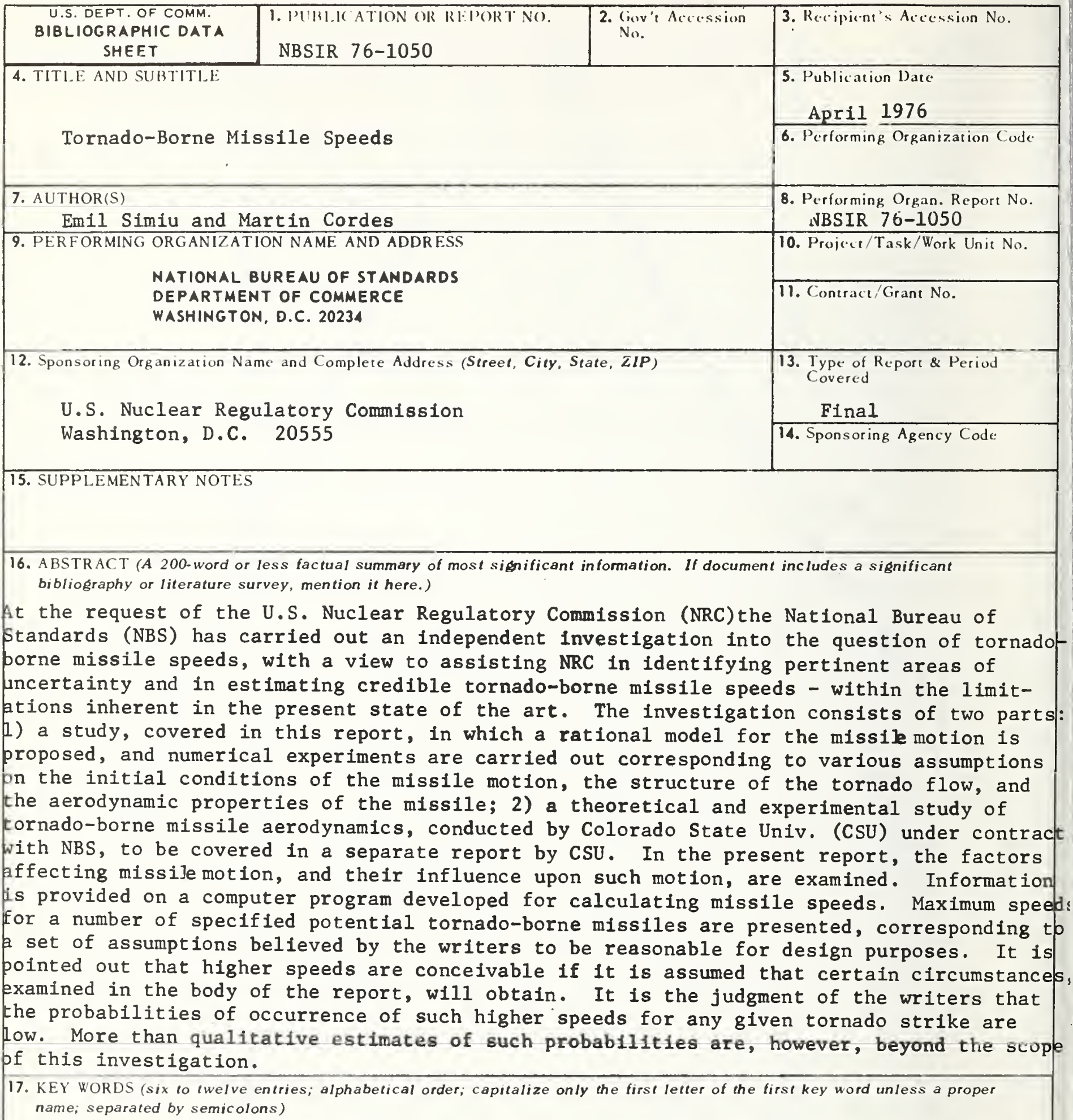

Missiles; nuclear engineering; structural engineering; tornadoes; wind.

18. AVAILABILITY $\quad \mathbf{X}$ Unlimited

For Official Distribution. Do Not Release to NTIS

Order From Sup. of Doc., U.S. Government Printing Office

Washington, D.C. 20402, SD Cat. No. C13

X Order From National Technical Information Service (NTIS) Springfield, Virginia 22151

\begin{tabular}{|l|c|}
\hline $\begin{array}{l}\text { 19. SECURITY CLASS } \\
\text { (THIS REPURT) } \\
\text { UNCL ASSIFIED }\end{array}$ & 21. NO. OF PAGES \\
\hline $\begin{array}{l}\text { 20. SECURITY CLASS } \\
\text { (THIS PAGE) } \\
\text { UNCLASSIFIED }\end{array}$ & $\begin{array}{c}22 . \text { PriCe } \\
\$ 4.50\end{array}$ \\
\hline
\end{tabular}





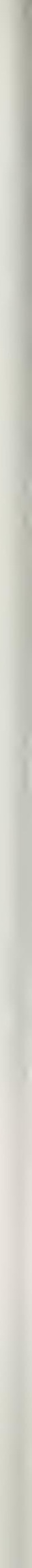

